\title{
Iterative Desensitisation of Image Restoration Filters under Wrong PSF and Noise Estimates
}

\author{
Miguel A. Santiago, ${ }^{1}$ Guillermo Cisneros, ${ }^{1}$ and Emiliano Bernués ${ }^{2}$ \\ ${ }^{1}$ Departamento de Señales, Sistemas y Radiocomunicaciones, Escuela Técnica Superior de Ingenieros de Telecomunicación, \\ Universidad Politécnica de Madrid, 28040 Madrid, Spain \\ ${ }^{2}$ Departamento de Ingeniería Electrónica y Comunicaciones, Centro Politécnico Superior, Universidad de Zaragoza, \\ 50018 Zaragoza, Spain
}

Received 19 July 2005; Revised 30 November 2006; Accepted 3 January 2007

Recommended by Bernard C. Levy

The restoration achieved on the basis of a Wiener scheme is an optimum since the restoration filter is the outcome of a minimisation process. Moreover, the Wiener restoration approach requires the estimation of some parameters related to the original image and the noise, as well as knowledge about the PSF function. However, in a real restoration problem, we may not possess accurate values of these parameters, making results relatively far from the desired optimum. Indeed, a desensitisation process is required to decrease this dependency on the parameter errors of the restoration filter. In this paper, we present an iterative method to reduce the sensitivity of a general restoration scheme (but specified to the Wiener filter) with regards to wrong estimates of the said parameters. Within the Fourier transform domain, a sensitivity analysis is tackled in depth with the purpose of defining a number of iterations for each frequency element, which leads to the aimed desensitisation regardless of the errors on estimates. Experimental computations using meaningful values of parameters are addressed. The proposed technique effectively achieves better results than those obtained when using the same wrong estimates in the Wiener approach, as well as verified on an SAR restoration.

Copyright (c) 2007 Miguel A. Santiago et al. This is an open access article distributed under the Creative Commons Attribution License, which permits unrestricted use, distribution, and reproduction in any medium, provided the original work is properly cited.

\section{INTRODUCTION AND BACKGROUND}

Let $\mathbf{h}$ be any generic two-dimensional degradation filter mask (PSF, usually invariant low-pass filter). Let $\mathbf{x}$ be an original image to be degraded. A generic linear shift-invariant degradation process of $\mathbf{x}$ using $\mathbf{h}$ can be written in a general way as

$$
\mathbf{y}=\mathbf{h} * * \mathbf{x}+\mathbf{n},
$$

where $\mathbf{y}$ is the degraded image (blurred and noisy image), and $\mathbf{n}$ is a two-dimensional matrix representing the added noise in the degradation. A restoration procedure will achieve a replica $\hat{\mathbf{x}}$ of the original image $\mathbf{x}$. The inversion of the degradation process cannot be derived directly; fundamentals on image processing [1-3] provide further details on this ill-posed problem. Therefore, a number of approaches have been investigated in the image restoration arena [4].

The classical stochastic regularisation method for image restoration minimises a global restoration error $\varepsilon$ by means of the function

$$
\varepsilon=\min \left(E\left\{\|\mathbf{y}-\hat{\mathbf{y}}\|^{2}\right\}\right),
$$

where $E\{\cdot\}$ represents the expectation operator.

Assuming circular convolution, as well as a stationary model for the blur $\mathbf{h}$, the original image $\mathbf{x}$, and the independent noise $\mathbf{n}$, the said minimisation provides an optimum linear solution written as a scalar operation for each 2D frequency component $\left(\omega_{i}, \omega_{j}\right)$ in the Fourier transform domain (using DFT) as

$$
\begin{aligned}
\hat{X}\left(\omega_{i}, \omega_{j}\right) & =G\left(\omega_{i}, \omega_{j}\right) Y\left(\omega_{i}, \omega_{j}\right) \\
& =\frac{H^{*}\left(\omega_{i}, \omega_{j}\right)}{\left|H\left(\omega_{i}, \omega_{j}\right)\right|^{2}+C\left(\omega_{i}, \omega_{j}\right)} Y\left(\omega_{i}, \omega_{j}\right)
\end{aligned}
$$

which is the Wiener restoration approach stood for the wellknown Wiener filter $G$ where $\hat{X}=\operatorname{DFT}(\widehat{\mathbf{x}}), Y=\operatorname{DFT}(\mathbf{y})$, 
$H=\operatorname{DFT}(\mathbf{h})$, and $C$ represents somehow an SNR parameter given by

$$
C\left(\omega_{i}, \omega_{j}\right)=\frac{S_{n n}\left(\omega_{i}, \omega_{j}\right)}{S_{x x}\left(\omega_{i}, \omega_{j}\right)},
$$

where $S_{x x}$ and $S_{n n}$ are the respective spectral densities of the original image $\mathbf{x}$ and the noise matrix $\mathbf{n}$.

On the basis of (3), the stochastic regularisation approach fully depends on a priori knowledge about $\mathbf{h}, \mathbf{x}$, and $\mathbf{n}$. Regarding $\mathbf{h}$, lots of work have been addressed to achieve estimates of the PSF, for example, [5-14]. On the other hand, common assumptions consider Gaussian noise for $S_{n n}$ and presume that the spectral density $S_{x x}$ of the unavailable original image $\mathbf{x}$ is not very different from the spectral density $S_{y y}$ of the degraded image $\mathbf{y}$, therefore $S_{x x} \cong S_{y y}$ [4]. However, it is important to point out other techniques for prior image modelling such as the use of Gauss-Markov random fields [15-18] or the wavelets models [19-21].

The more correct those estimates are, the closer the restoration result of (3) is to the optimum solution of (2). Nevertheless, the sensitivity of (3) to wrong estimates is very high; for example, relatively small deviations from the real (unknown) value of $C$ make (3) yield results very far from the desired optimum. State-of-art provides some algorithms of robust filters [22-24], particularly addressed to obtain good results in spite of the presence of outliers within the noise (when expected to be Gaussian). Nonetheless, our objective is not to obtain an independent filter, but to improve the results of an original restoration (relative procedure) when having wrong estimates of the dependant parameters. That is to say, we aim to provide the original restoration with robustness in a parametrical sense by means of a desensitisation process. Additionally, a large literature can be found regarding researches on iterative restoration (e.g., [25-32]) as an alternative to solve this problem.

In order to simplify notation, the reference to the element $\left(\omega_{i}, \omega_{j}\right)$ of the matrices in the frequency domain will be removed from all formulae throughout the remainder of this paper. Besides, it must be taken into account that all mathematical expressions involving matrices in the Fourier transform domain will be scalar computations for each frequency component $\left(\omega_{i}, \omega_{j}\right)$.

Moreover, since we use estimates of the parameters in the restoration side, let us remark them by including a suffix $e$ all along the analysis to differ from real values, that is, $H_{e}$ and $C_{e}$ for the Wiener approach.

In short, Section 2 proposes an iterative model for desensitisation with respect to the before-mentioned estimates. Afterwards, Section 3 provides an analysis on the degree of desensitisation achieved, as well as a proposal for the number of iterations. Finally, Section 4 offers some restoration results to present the successful benefits reached by our innovative restoration scheme.

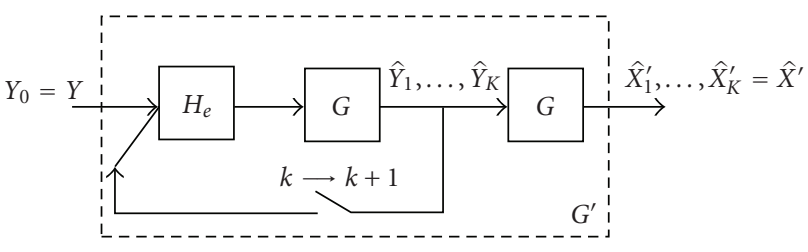

Figure 1: Proposed restoration scheme.

\section{RESTORATION MODEL}

In the light of the above, we can write the restored image (Fourier transform) in a general way as

$$
\widehat{X}=G Y=G(H X+N)=G H X+G N,
$$

where $N=\operatorname{DFT}(\mathbf{n})$. Going a step further, our research aims to build an innovative restoration filter $G^{\prime}$ based on $G$ whose sensitivity with respect to the estimates related to the restoration model (such as $H_{e}$ and $C_{e}$ in the Wiener approach) is smaller than that of $G$. This filter $G^{\prime}$ will provide another replica $\hat{\mathbf{x}}^{\prime}$ of the original image, whose Fourier transform $\hat{X}^{\prime}=\operatorname{DFT}\left(\hat{x}^{\prime}\right)$ can be written as

$$
\hat{X}^{\prime}=G^{\prime} Y=G^{\prime}(H X+N)=G^{\prime} H X+G^{\prime} N .
$$

In order to achieve this purpose, $G^{\prime}$ is defined by applying an iterative process of degradations and restorations, using $H_{e}$ and $G$, respectively. This process is graphically explained in Figure 1.

The input at any iteration $k(k=1,2, \ldots, K)$ is an image $\mathbf{y}_{k-1}\left(Y_{k-1}=\operatorname{DFT}\left(\mathbf{y}_{k-1}\right)\right)$ where $Y_{0}=Y=H X+N$ (i.e., to say, the degraded image $\mathbf{y})$. The corresponding output is an approach $\widehat{\mathbf{x}}_{k}^{\prime}$ to $\widehat{\mathbf{x}}^{\prime}\left(\hat{X}_{k}^{\prime}=\operatorname{DFT}\left(\hat{x}_{k}^{\prime}\right)\right)$. After the last iteration $K$, we will have $\hat{X}^{\prime}$ of (6) as $\hat{X}^{\prime}=\hat{X}_{K}^{\prime}$. A criterion will be adopted to define this total number of iterations $K$.

Actually, this proposed restoration method is applied within the Fourier transform domain on the degraded spectrum $Y$ and, as stated later, the number of iterations $K$ is a function of each frequency element, as denoted by the inclusion of the symbol $\left(\omega_{i}, \omega_{j}\right)$ in the restoration scheme.

Mathematically, the iterative process of Figure 1 is explained for every frequency pair as follows:

$$
\begin{array}{rlrl}
\hat{Y}_{1}=G H_{e} Y_{0}=G H_{e} Y & \hat{X}_{1}^{\prime}= & G \hat{Y}_{1}=G\left(G H_{e}\right) H X \\
& =G H_{e}(H X+N) & & \\
& & G\left(G H_{e}\right) N \\
\hat{Y}_{2}=G H_{e} \hat{Y}_{1} & \hat{X}_{2}^{\prime}= & G \hat{Y}_{2}=G\left(G H_{e}\right)^{2} H X \\
=\left(G H_{e}\right)^{2}(H X+N) & & +G\left(G H_{e}\right)^{2} N
\end{array}
$$




$$
\begin{aligned}
& \widehat{Y}_{3}=G H_{e} \hat{Y}_{2} \\
& =\left(G H_{e}\right)^{3}(H X+N) \\
& \hat{Y}_{k}=G H_{e} \hat{Y}_{k-1} \\
& =\left(G H_{e}\right)^{k}(H X+N) \\
& \widehat{Y}_{K}=G H_{e} \hat{\hat{Y}}_{K-1} \\
& =\left(G H_{e}\right)^{K}(H X+N) \\
& \hat{X}_{3}^{\prime}=G \hat{Y}_{3}=G\left(G H_{e}\right)^{3} H X \\
& +G\left(G H_{e}\right)^{3} N \\
& \hat{X}_{k}^{\prime}=G \hat{Y}_{k}=G\left(G H_{e}\right)^{k} H X \\
& +G\left(G H_{e}\right)^{k} N \\
& \hat{X}_{K}^{\prime}=G Y_{K}=G\left(G H_{e}\right)^{K} H X
\end{aligned}
$$

By comparing (6) with any row (right side) of (7), we can write our proposed desensitisation filter $G^{\prime}$ at any iteration $k$ and for each frequency element $\left(\omega_{i}, \omega_{j}\right)$ as

$$
G^{\prime}=G\left(G H_{e}\right)^{k} \text {. }
$$

Having a look to (8), we can verify the dependency of the new filter $G^{\prime}$ on three basic parameters such as the original restoration filter $G$ (e.g., the Wiener approach), the regularisation product $G H_{e}$ (different from the original regularisation $G H)$ as explained in the restoration regularisation theory [33-35], and the number of iterations $k$ of the model shown in Figure 1.

Therefore, our goal now aims to demonstrate the desensitisation behaviour of our proposed restoration filter $G^{\prime}$, showing which conditions lead to successful results, purposely, the total number of iterations $K$ applied to each pair $\left(\omega_{i}, \omega_{j}\right)$. A first approach to this idea was initially coped with in [36] where some preliminary results meant opening steps to the current fully study throughout this paper.

\section{SENSITIVITY OF THE FILTERS}

\subsection{Condition establishment}

Let us now compute and compare the sensitivities of $G$ and $G^{\prime}$ with respect to the estimates and assumptions required in the restoration process. Let $S_{G}$ be the sensitivity regarding the filter $G$ which can be defined as

$$
S_{G}=\frac{\partial G}{\partial P_{1}} d P_{1}+\frac{\partial G}{\partial P_{2}} d P_{2}+\cdots+\frac{\partial G}{\partial P_{n}} d P_{n},
$$

where $P_{1}, P_{2}, \ldots, P_{n}$ are the parameters to be estimated in the restoration model. For instance, $H_{e}$ and $C_{e}$ stand for the required estimates in the Wiener restoration method within the Fourier domain which involve the before-mentioned parameters in the introductory section, explicitly, the PSF function $\mathbf{h}\left(H_{e}\right)$ and the original image $\mathbf{x}$, and the noise $\mathbf{n}\left(C_{e}\right)$. Indeed, this Wiener approach will be coped with in the remainder of this paper in order to present both mathematical analysis and computed results. Hence, we can rewrite (9) as

$$
S_{G}=\frac{\partial G}{\partial H_{e}} d H_{e}+\frac{\partial G}{\partial C_{e}} d C_{e}
$$

Analogously, the sensitivity concerning the proposed filter $G^{\prime}$ can be expressed as follows:

$$
S_{G}^{\prime}=\frac{\partial G^{\prime}}{\partial H_{e}} d H_{e}+\frac{\partial G^{\prime}}{\partial C_{e}} d C_{e}
$$

Multiplying and dividing (11) by $\partial G^{\prime}$, both sensitivities (10) and (11) can be related to each other as

$$
S_{G}^{\prime}=\frac{\partial G^{\prime}}{\partial G}\left(\frac{\partial G}{\partial H_{e}} d H_{e}+\frac{\partial G}{\partial C_{e}} d C_{e}\right)=\frac{\partial G^{\prime}}{\partial G} S_{G}
$$

After differentiating the filter $G^{\prime}$ with respect to $G$ taking the expression (8) into account, we come up with a milestone concept within our research into restoration sensitivity, namely, the relative sensitivity function of $G^{\prime}$ regarding $G$ for a given pair $\left(\omega_{i}, \omega_{j}\right)$ denoted by $Z(k)$ whose definition can be described as

$$
Z(k)=\frac{S_{G}^{\prime}}{S_{G}}=\frac{\partial G^{\prime}}{\partial G}=\frac{\partial}{\partial G}\left[G\left(G H_{e}\right)^{k}\right]=(k+1)\left(G H_{e}\right)^{k}
$$

Consequently, we find the condition for the proposed filter $G^{\prime}$ to be less sensitive than $G$ with regards to wrong assumptions of $H_{e}$ and wrong estimates of $C_{e}$ as

$$
S_{G}^{\prime}<S_{G} \Longleftrightarrow Z(k)<1
$$

As a corollary, this condition (14) can be extended to not only a global sensitivity study but also a focusing of the analysis on a particular estimation of the restoration model regardless of which one is considered. Thus, taking (9) into consideration, let us define the sensitivity of the filter $G$ with respect to the parameter $P$ as $S_{G}^{P}$,

$$
S_{G}^{P}=\frac{\partial G}{\partial P}
$$

Comparing both sensitivities $S_{G}^{\prime}$ and $S_{G}^{P}$ yields

$$
\frac{S_{G^{\prime}}^{\prime P}}{S_{G}^{P}}=\frac{\partial G^{\prime} / \partial P}{\partial G / \partial P}=\frac{\partial G^{\prime}}{\partial G}=Z(k)
$$

Hence, this leads to the conclusion stated by the corollary

$$
S_{G}^{\prime}<S_{G} \Longleftrightarrow Z(k)<1 \Longleftrightarrow S_{G^{\prime}}^{P}<S_{G}^{P}
$$

applied to whatsoever parameter of the restoration approach, particularly, $H_{e}$ and $C_{e}$ within our Wiener method.

\subsection{Condition analysis}

As a first step of our analysis, let us consider the regularisation term $G H_{e}$ involved in the expression (13). In view of (3), this product can be rewritten as

$$
G H_{e}=\frac{H_{e}^{*} H_{e}}{H_{e}^{*} H_{e}+C_{e}}=\frac{\left|H_{e}\right|^{2}}{\left|H_{e}\right|^{2}+C_{e}} .
$$




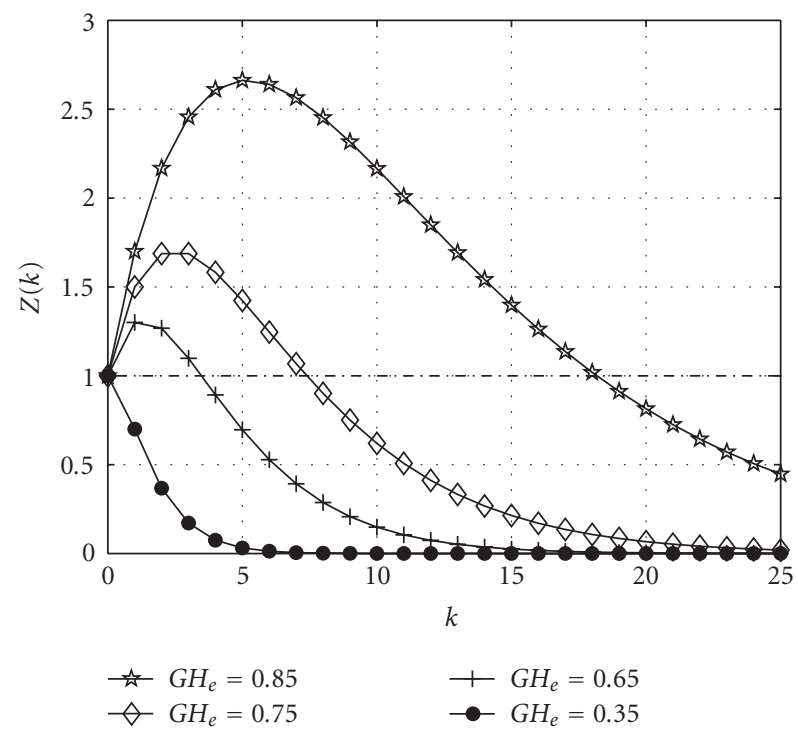

FIgURE 2: Relative sensitivity function $Z(k)$.

By definition, in the presence of noise, that is to say, real restoration conditions,

$$
\left.S_{n n}\right|_{e}>0,\left.\quad S_{x x}\right|_{e} \geq 0 \Longrightarrow C_{e}=\frac{\left.S_{n n}\right|_{e}}{\left.S_{x x}\right|_{e}}>0 \quad \forall\left(\omega_{i}, \omega_{j}\right)
$$

Taking for granted that $\left|H_{e}\right|^{2} \geq 0$ and combining (19) into (18), the product $G H_{e}$ can be ranged as follows:

$$
0 \leq G H_{e}<1 \Longrightarrow 0 \leq\left(G H_{e}\right)^{k} \leq G H_{e}<1 \quad \forall\left(\omega_{i}, \omega_{j}\right), \forall k \geq 1 .
$$

As a result of (20), we can conclude that the relative sensitivity function $Z(k)=(k+1)\left(G H_{e}\right)^{k}$ of $(13)$ is not either monotonically increasing or decreasing with the number of iterations $k$, but it may show a relative maximum extreme, depending on the value of the term $G H_{e}$ for a particular pair $\left(\omega_{i}, \omega_{j}\right)$. This is illustrated in Figure 2 for several regularisation values.

From the last plot, we find the expected maximum extremes of $Z(k)$ as peaks located on specific numbers of iterations $k$ depending on which regularisation value $G H_{e}$ is considered. Clearly, the lower the product $G H_{e}$ is, the less iterations $k$ are required to reach the consequent less intensified maximum of $Z(k)$. Furthermore, high enough regularisation conditions (i.e., to say, low values of $G H_{e}$ ) make $Z(k)$ fully decreasing monotonic.

Nonetheless, the main conclusion to be drawn from Figure 2 is related to the sensitivity condition (14), once imposing an identity $Z(k)$-level over the graphic, which shows the iteration from which the appointed desensitisation is achieved. In fact, looking at the plot, we can say that regardless of the value of the product $G H_{e}, G^{\prime}$ is less sensitive than $G$ if the number of iterations $k$ is high enough. Under this hypothesis, we may increase the value of $k$ as much as wished in order to prevent poor restoration results under wrong estimates of the implied parameters $\left(H_{e}\right.$ and $\left.C_{e}\right)$. Unfortunately, this statement is not true since there are other restoration factors to be considered. Precisely, next section deals with this issue.

\subsection{Condition limits}

The goal of this section is to analyse the proposed filter $G^{\prime}$ from a view based on the restoration error in order to verify how the desensitisation influences the final results. Thus, let $E_{t}$ be the Fourier Transform of the restoration error with regards to our proposed model whose expression is

$$
E_{t}^{\prime}=\hat{X}^{\prime}-X
$$

Besides, the digital image theory [1-3] divides the restoration error into two meaningful components as follows:

$$
E_{t}^{\prime}=E_{r}^{\prime}+E_{n}^{\prime}
$$

where $E_{r}^{\prime}$ and $E_{n}^{\prime}$ are the well-known image-dependent and noise-dependent components in the Fourier domain, respectively.

By taking (6) into account and comparing both expressions (21) and (22), it leads to

$$
\left(G^{\prime} H X+G^{\prime} N\right)-X=E_{r}^{\prime}+E_{n}^{\prime}
$$

Consequently, we come up with the definitions of the restoration error components as

$$
E_{r}^{\prime}=\left(G^{\prime} H-I\right) X, \quad E_{n}=G^{\prime} N
$$

where $I$ represents the identity matrix for every pair $\left(\omega_{i}, \omega_{j}\right)$.

Analogously, we can rewrite the same expressions regarding the original restoration filter $G$ (Wiener approach) as below:

$$
E_{r}=(G H-I) X, \quad E_{n}=G N .
$$

However, we are actually interested in contrasting the restoration errors from both models in order to demonstrate the influence of the desensitisation on the restored image. Hence, let $\delta_{r}$ and $\delta_{n}$ be the relative image-dependent and noise-dependent errors, respectively, as

$$
\delta_{r}=\frac{E_{r}^{\prime}}{E_{r}}, \quad \delta_{n}=\frac{E_{n}^{\prime}}{E_{n}} .
$$

Substituting (24), (25) into (26), in addition to applying the definition of our filter $G^{\prime}(8)$, we have

$$
\begin{gathered}
\delta_{r}(k)=\frac{\left[G\left(G H_{e}\right)^{k} H-I\right] X}{(G H-I) X}=\frac{1-(G H)\left(G H_{e}\right)^{k}}{1-G H}, \\
\delta_{n}(k)=\frac{E_{n}^{\prime}}{E_{n}}=\frac{G\left(G H_{e}\right)^{k} N}{G N}=\left(G H_{e}\right)^{k}
\end{gathered}
$$




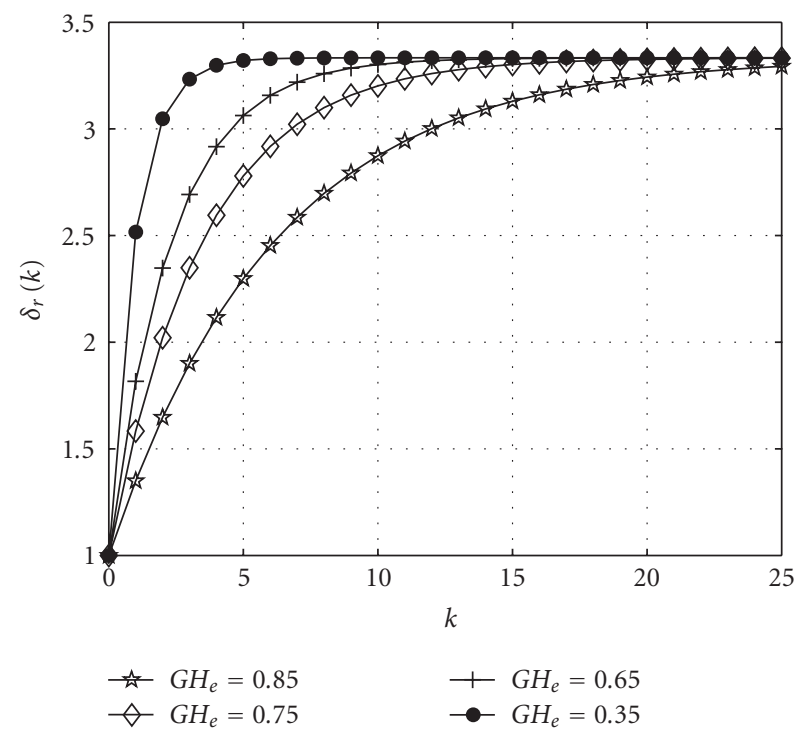

FIgURE 3: Relative image-dependent error $\delta_{r}(k)$.

whose plots with respect to the number of iterations $k$ are illustrated in Figures 3 and 4 using the same regularisation values $G H_{e}$ as in Figure 2 and holding fixed the original product $\mathrm{GH}$ to 0.7 .

Looking at those figures, we find out the mentioned constraint in the last section which prevented increasing unboundedly the number of iterations in order to intensify the desensitisation level as shown in Figure 2. The more we raise the value of $k$, the higher the relative image-dependent error $\delta_{r}$ and, on the contrary, the lower the relative noisedependent error $\delta_{n}$ becomes.

Consequently, we are forced to strike a trade-off between both component errors whether successful desensitisation results are pretended for a specific value of iterations, besides taking the condition (14) into account.

As a matter of interest, it can be easily demonstrated by applying the range (20) to the expressions (27), apart from assuming that the original regularisation $G H$ also fulfills that range, then,

$$
\begin{gathered}
\delta_{r}(k) \geq 1 \quad \forall\left(\omega_{i}, \omega_{j}\right), \forall k \geq 1, \\
0 \leq \delta_{n}(k)<1 \quad \forall\left(\omega_{i}, \omega_{j}\right), \forall k \geq 1
\end{gathered}
$$

which states that the noise-dependent error is always lower for our proposed restoration model than that of the original schema (Wiener approach). Conversely, the imagedependent error becomes higher giving an evidence of a much better improvement on very noisy degraded images than those corrupted by other kind of degradations.

Going a step further, it is important to point out that the condition (28) is not always satisfied if the said hypothesis regarding $G H$ is not kept. Indeed, when wrong estimates about the PSF are considered, this product can be over the unity or even negative making the relative image-dependent error $\delta_{r}$ decrease with the number of iterations $k$. Although it seems to be another successful result, however, it is not likely

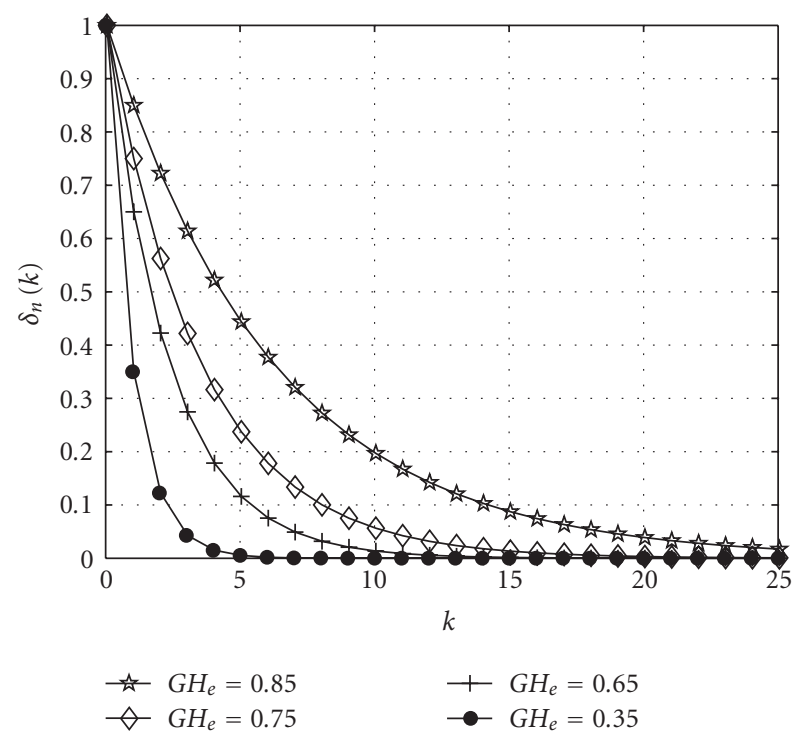

FIgURE 4: Relative noise-dependent error $\delta_{n}(k)$.

to have this situation too expanded all along the spectrum when reasonable estimates of $H_{e}$ are taken, but if so, the benefits obtained by reducing the image-dependent error are not enough to improve the extreme impairments caused by the high deviation from the real value of $H$.

\subsection{Recommended number of iterations}

Following the basis on our research, we cope with the task of working out an appropriate number of iterations $K$ applied to the proposed model. Let us remind that we are using scalar computations of matrices in the Fourier domain and, consequently, the obtained number of iterations will be a function of every pair $\left(\omega_{i}, \omega_{j}\right)$.

As a result of previous sections, we can see that the increase of the number of iterations $k$ may provide a less sensitive restoration filter $G^{\prime}$ as desired. Nevertheless, both the image-dependent and noise-dependent restorations errors do not allow raising it unboundedly. Thus, we will try to find a required trade-off.

From the beginning, our goal is to reduce the value of the relative sensitivity function $Z(k)$ as stated in condition (14). Since this function does not provide any minimum as illustrated in Figure 2, let us optimise another $Z(k)$ property which fulfills our desensitisation purpose. With this in mind, let us look for a maximum of efficiency for the incremental complexity introduced in the restoration process by increasing the number of iterations from $k$ to $k+1$. In other words, let us seek a value of $k$ from which we do not get much more improvements on desensitisation but, on the contrary, the complexity is notably incremented.

The next step consists of giving a mathematical sense to this conceptual criterion with regards to $Z(k)$. Knowing that we can simulate the variation of a function by means of its derivative, the reduction of sensitivity can be accomplished through the first derivative of $Z(k)$, namely, $Z^{\prime}(k)$. In view 


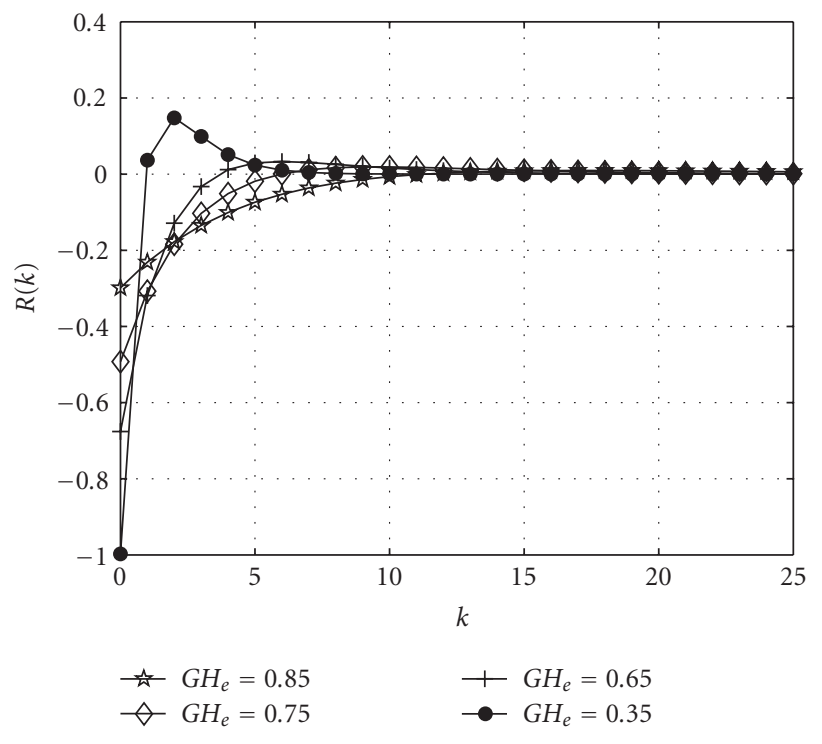

FIGURE 5: Function $R(k)$ defined as the second derivative of $Z(k)$.

of the fact that the desensitisation change is expected to be maximised, the second derivative of $Z(k)$ is herein the aimed function denoted by $R(k)$,

$$
R(k)=Z^{\prime \prime}(k)=\frac{\partial^{2} Z(k)}{\partial k^{2}} .
$$

After some calculations (see Appendix A), we obtain the definition of $R(k)$,

$$
R(k)=\left(G H_{e}\right)^{k} \ln \left(G H_{e}\right)\left[2+(k+1) \ln \left(G H_{e}\right)\right]
$$

whose representation, as illustrated in Figure 5, gives us a full evidence of the successful approach due to the presence of maximum extremes.

Therefore, our proposed desensitisation criterion can be summarized as the value of $k$ which fulfills

$$
\max [R(k)], \quad Z(k)<1 \quad \forall k \geq 1 .
$$

In Appendix B, it is further demonstrated that the solved number of iterations $K$ can be expressed as follows:

$$
K=\operatorname{round}\left\{-\left[1+\frac{3}{\ln \left(G H_{e}\right)}\right]\right\}
$$

subject to a constraint on the regularisation term $G H_{e}$,

$$
0.14<G H_{e}<0.84 \text {. }
$$

With the purpose of making sure about the successful criterion, let us present numeric results by means of Table 1 which comes together all the mainly showed concepts such as $G H_{e}, K, Z(k), \delta_{r}(k)$, and $\delta_{n}(k)$ (relative errors values are in $\mathrm{dB}$ ), leaving the original regularisation $G H$ unalterable to the value 0.7 . Looking at this table, we can see that the improvements achieved for $\delta_{n}(k)$ are greater than the impairments obtained from $\delta_{r}(k)$, always satisfying the desensitisation condition $Z(k)<1$. For that reason, it is expected to have good restoration results with a rough estimation of noise in a very wide range, much better than the other kind of wrong estimates.

\section{SIMULATION RESULTS}

With the intention of proving the successful benefits achieved by our innovative restoration model, let us simulate some illustrative examples. Purposely, the image selected for testing is the well-known Cameraman $256 \times 256$ sized making easier to compare the obtained results with those from other researches in the restoration area.

As stated in Section 1, the original image is disturbed by a degradation filter and an additive noise. In order to show a variety of meaningful examples, let us make use of several common filters within the application of astronomical imaging such as the motion blur, the atmospheric turbulence degradation (Gaussian), and the uniform blur. Moreover, both the most typical Gaussian white noise and other more complicated artefacts such as "salt and pepper" or multiplicative noises (speckle) are added to the blurred image. Thereby, the next subsections aim to specify the proposed restoration method by collecting all these possible options in such a way that the main goals of our paper can be clearly evidenced, that is to say, the improvements accomplished by our iterative scheme $G^{\prime}$ on an original restoration filter $G$ when wrong estimates of the parameters are considered.

Regarding the restoration filter $G$, as indicated throughout the paper, the minimum mean-squared method (Wiener filter) is used and, consequently, $H_{e}$ and $C_{e}$ are the parameters to be estimated. Let us remind that they represent the frequency estimates of the three generic restoration parameters: the original image and the noise $\left(C_{e}\right)$ and the degradation filter $\left(H_{e}\right)$.

In view of the fact that those parameters must be altered to show the efficacy of the desensitised filter $G^{\prime}$, let us arrange some guidelines to modify each one. Firstly, we take into consideration the said assumption pointed out in Section 1 about the original image whose spectral density $S_{x x}$ is roughly approximated by that of the degraded image $S_{y y}$. Concerning the noise, we assume a Gaussian estimation whose variance stands for the parameter to be altered. Consequently, the value of $C_{e}$ in (4) changes from the real one. Finally, we consider a motion blur for the degradation estimation $H_{e}$ modifying the inclination parameter and the number of moved pixels. Furthermore, we deal with not only the selection of the same category of input processes, that is to say, gaussian noise and motion blur as real values, but also with providing other classes such as commented at the beginning of this section.

By means of a relative error, we manage to measure the deviations from the real value of those parameters. Thus, let $\varepsilon_{P}$ be the relative error of a generic parameter $P$ defined as follows:

$$
\mathcal{E}_{P}=\frac{P_{\text {real }}-P_{\text {estimated }}}{P_{\text {real }}} \cdot 100,
$$

where $P_{\text {real }}$ and $P_{\text {estimated }}$ stand for the respective real and estimated values of the parameter $P$. 
TABLE 1: Numeric results for the functions $G H_{e}, K, Z(k=K), \delta_{r}(k=K)$, and $\delta_{n}(k=K)$ applied to the desensitisation.

\begin{tabular}{c|rrrrrrrrrrrrr}
\hline$G H$ & 0.20 & 0.25 & 0.30 & 0.35 & 0.40 & 0.45 & 0.50 & 0.55 & 0.60 & 0.65 & 0.70 & 0.75 & 0.80 \\
\hline$K$ & 1 & 1 & 1 & 2 & 2 & 3 & 3 & 4 & 5 & 6 & 7 & 9 & 12 \\
$Z(k=K)$ & 0.40 & 0.50 & 0.60 & 0.37 & 0.48 & 0.36 & 0.50 & 0.46 & 0.47 & 0.53 & 0.66 & 0.75 & 0.89 \\
$\delta_{r}(k=K)$ & 9.15 & 8.79 & 8.41 & 9.68 & 9.43 & 9.89 & 9.66 & 9.88 & 9.97 & 9.99 & 9.94 & 9.99 & 10.03 \\
$\delta_{n}(k=K)$ & -13.98 & -12.04 & -10.46 & -18.24 & -15.92 & -20.81 & -18.06 & -20.77 & -22.18 & -22.45 & -21.69 & -22.49 & -23.26 \\
\hline
\end{tabular}

Let us remark that this relative error is not directly addressed to the complex and two-dimensional parameters $H_{e}$ and $C_{e}$, but applied on other dependent variables such as the blurring inclination $\theta$ or the noise variance $\sigma^{2}$ as previously mentioned. Provided that these parameters are real variables, the relative error $\varepsilon_{P}$ is also extended along the range $-\infty<\varepsilon_{P}<+\infty$, even though we only consider the significant values ranged between -100 and $100 \%$.

In order to properly show the steps up, the results are always presented with regards to the Wiener filter when using optimum estimates; the same when wrong estimates are taking into account and, finally, by applying our restoration model under the same mistaken estimates.

Let us remind that the proposed desensitisation mechanism yields a different number of iterations for every pair $\left(\omega_{i}, \omega_{j}\right)$ due to its dependence on the product $G H_{e}$, which is, likewise, variable with each frequency component, namely, $K\left(\omega_{i}, \omega_{j}\right)=K\left[G H_{e}\left(\omega_{i}, \omega_{j}\right)\right]$. By using the expression of (33), we obtain a value of $K$ for those pairs whose related regularisation term $G_{e}$ is within the range given by (34). Thus, a criterion will be adopted for choosing a number of iterations for the rest of frequencies. Owing to the increasing trend of $K$ with respect to $G H_{e}$ (see Table 1), all pairs whose corresponding regularisation value exceeds 0.84 are associated to a constant number of iterations, equal to the maximum value of $K$ reached by those within the range. Respectively, the minimum value of $K$ computed within the range is applied to those under 0.14 , explicitly, no iterations are brought into play.

Eventually, a way to numerically contrast the restoration results is obtained by an image quality parameter named as the improvements on the signal-to-noise ratio, that is, ISNR,

$$
\operatorname{ISNR}=10 \log \left\{\frac{\sum_{i=0}^{M-1} \sum_{j=1}^{N-1}[x(i, j)-y(i, j)]^{2}}{\sum_{i=0}^{M-1} \sum_{j=1}^{N-1}[x(i, j)-\hat{x}(i, j)]^{2}}\right\},
$$

where $x(i, j), y(i, j)$, and $\hat{x}(i, j)$ represent the $M \times N$ sized images $\mathbf{x}, \mathbf{y}$, and $\hat{\mathbf{x}}$, respectively. The more similar the restored image $\hat{\mathbf{x}}$ is to the original image $\mathbf{x}$, the higher the parameter ISNR becomes.

Example 1. In a first simulation, we investigate the case where wrong estimates of the parameter $C_{e}$ are considered and the value of $H_{e}$ is not altered with regards to $H$.

We start applying a motion blur to the original image described by a length of 15 pixels and an angle of 45 degrees in a counter-clockwise direction. Later on, a Gaussian noise is added following a blurred signal-to-noise ratio BSNR ranged between 0 and $30 \mathrm{~dB}$.
In the restoration process, we keep the parameter $H_{e}$ taking the same values of the original motion blur. On the other hand, apart from the fixed error result of the original image estimation $\left.S_{x x}\right|_{e} \cong S_{y y}$, the parameter $C_{e}$ is distorted by changes in the variance of an estimated Gaussian noise. Expressly, we evaluate the variations of this parameter using the relative error of the standard deviation $\sigma$ associated to the noise, namely, $\varepsilon_{\sigma}$ whose expression can be written using (35) as

$$
\varepsilon_{\sigma}=\frac{\sigma_{\text {real }}-\sigma_{\text {estimated }}}{\sigma_{\text {real }}} \cdot 100 .
$$

After solving this equation regarding $\sigma_{\text {estimated }}$,

$$
\sigma_{\text {estimated }}=\sigma_{\text {real }}\left(1-\frac{\varepsilon_{\sigma}}{100}\right),
$$

and replacing the standard deviation $\sigma$ with the squared associated variance $\sigma^{2}$, we can express the estimated variance as follows:

$$
\sigma_{\text {estimated }}^{2}=\left[\sqrt{\sigma_{\text {real }}^{2}}\left(1-\frac{\varepsilon_{\sigma}}{100}\right)\right]^{2} .
$$

On the way to achieve a significant range of results, we alter the estimated noise variance (39) so far as the error $\varepsilon_{\sigma}$ covers the values between -100 and $100 \%$. Hence, we design a set of representations with the distribution of ISNR obtained by both the Wiener filter $G$ and our desensitised restoration filter $G^{\prime}$, when $\sigma_{\text {estimated }}^{2}$ is modified in relation to $\varepsilon_{\sigma}$ within the said range. Specifically, we can find these illustrations in Figures 6(a), 6(b), 6(c), and 6(d) for different values $\sigma_{\text {real }}^{2}$ indicated by an BSNR of $0,10,20$, and $30 \mathrm{~dB}$. Besides, a horizontal line is included symbolizing the constant value of ISNR reached when optimum estimates (real values) are considered in the Wiener filter.

Having a look to those figures, let us define the target area as the range of $\varepsilon_{\sigma}$ where the value of ISNR obtained by the filter $G^{\prime}$ exceeds that of the Wiener approach $G$. Thus, we appreciate how wider this region becomes as we decrease the input BSNR. If we are located in the positive side of $\varepsilon_{\sigma}$, that is to say, $\sigma_{\text {estimated }}^{2}<\sigma_{\text {real }}^{2}$ as derived from (39), the percentage of error needed to reach the target region goes down as the BSNR is reduced, even being fully target area when an enough noise level is applied, for instance, $10 \mathrm{~dB}$. Alternatively, in the negative side of $\varepsilon_{\sigma}$, explicitly, $\sigma_{\text {estimated }}^{2}>\sigma_{\text {real }}^{2}$, the value of ISNR got by the desensitised restoration is barely greater than that of the Wiener filter excluding high enough noise conditions $(10 \mathrm{~dB})$, where the target area precisely extends to all the positive values of $\varepsilon_{\sigma}$. 


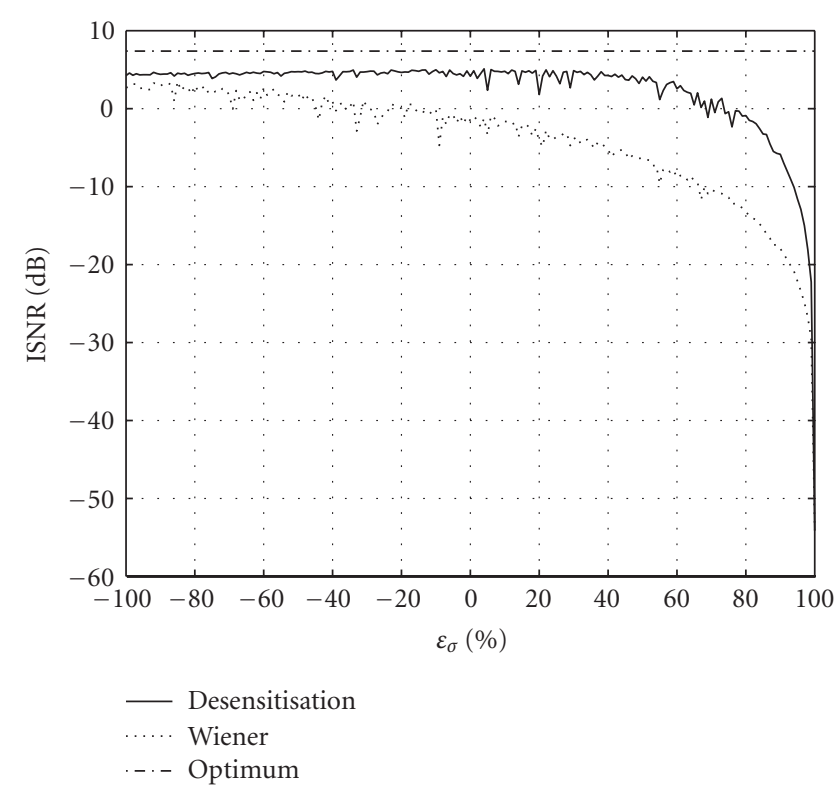

(a)

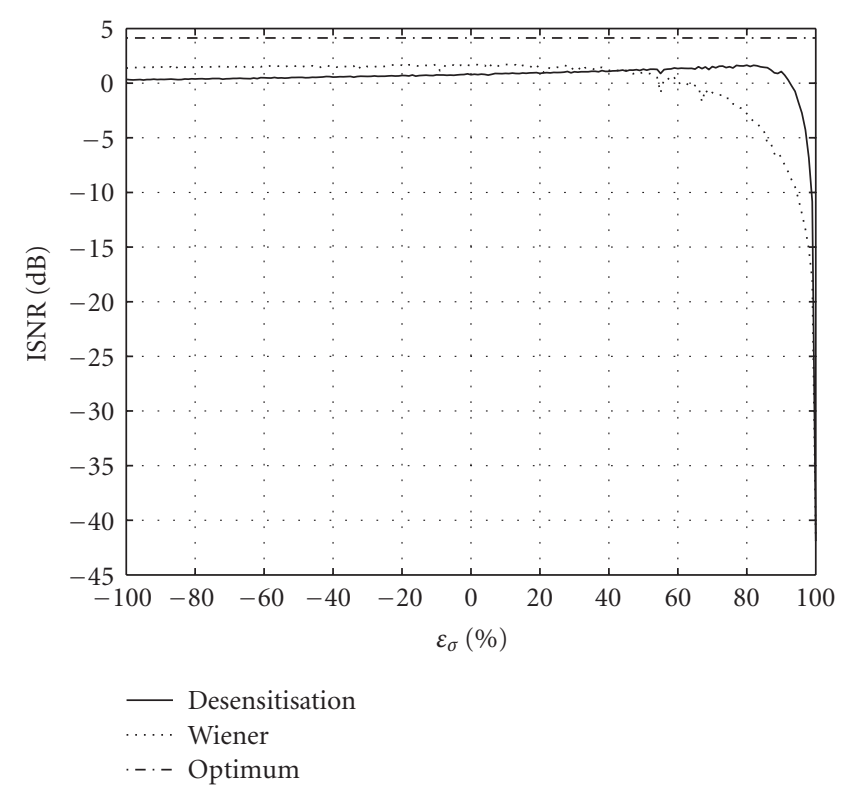

(c)

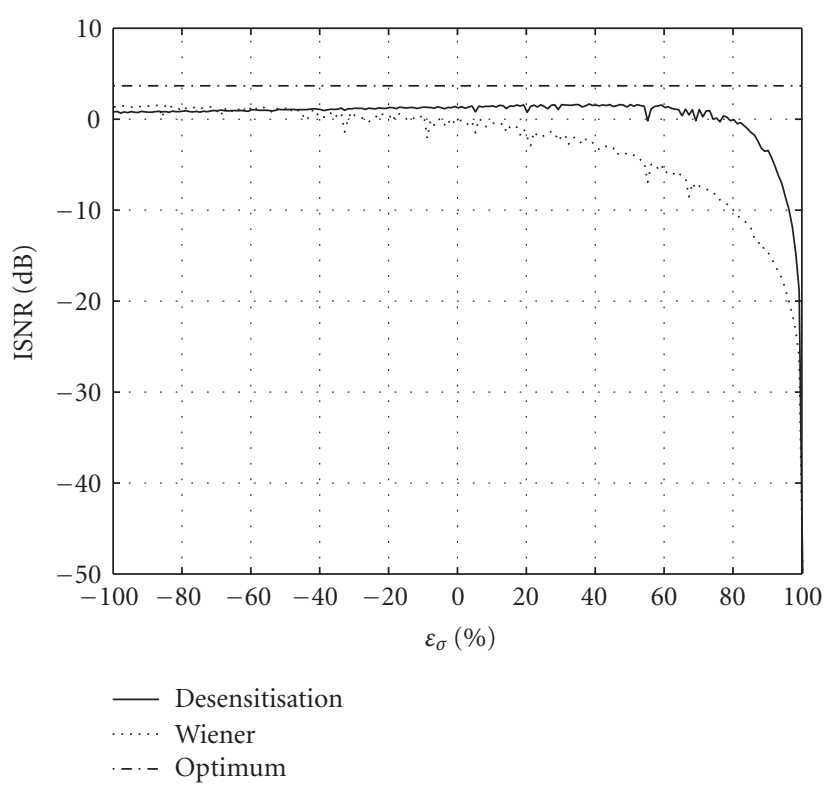

(b)

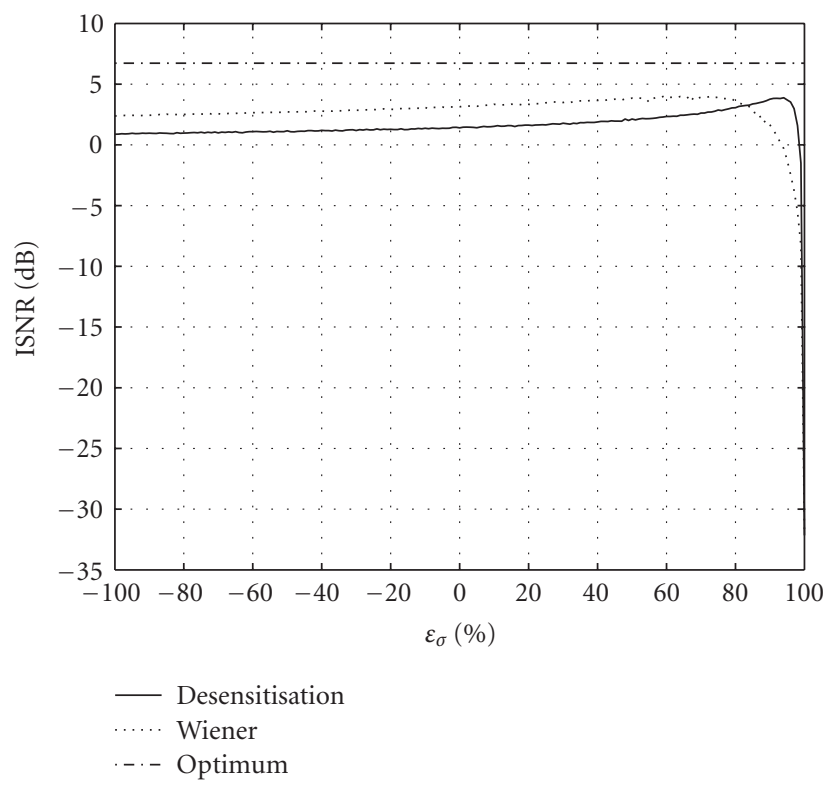

(d)

FIGURE 6: Distributions of ISNR obtained by both the Wiener filter and our desensitised method when the estimated Gaussian noise variance is altered according to a relative error $\varepsilon_{\sigma}$ leaving the PSF estimation unchanged (motion blur). Different noise levels are applied in relation to a BSNR of (a) $0 \mathrm{~dB}$, (b) $10 \mathrm{~dB}$, (c) $20 \mathrm{~dB}$, and (d) $30 \mathrm{~dB}$. Besides, a horizontal line is included symbolizing the constant value of ISNR reached when optimum estimates are considered in the Wiener filter.

Therefore, we can conclude that noise conditions rationally influence values of the relative error $\varepsilon_{\sigma}$ which are minimally required to get successful results with our proposed scheme. Moreover, estimates of variance $\sigma_{\text {estimated }}^{2}$ under the real values $\sigma_{\text {real }}^{2}$ are more likely to be in the target region than those estimates which are over the real ones.
Paying attention again to Figure 6, we notice a parabolic shape of every distribution ISNR which decreases towards the relative error of $100 \%\left(\sigma_{\text {estimated }}^{2}=0\right)$. Furthermore, the desensitised filter makes this parabola more constant leaving the declining point at a higher positive $\varepsilon_{\sigma}$. 


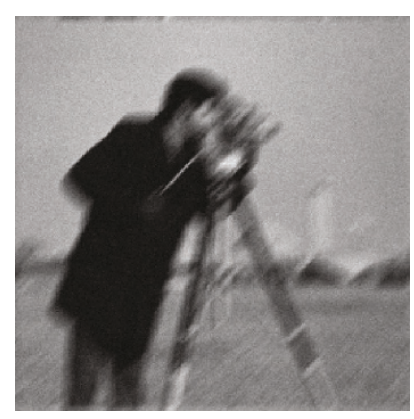

(a)

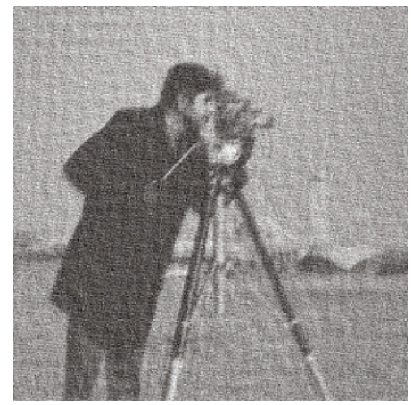

(c)

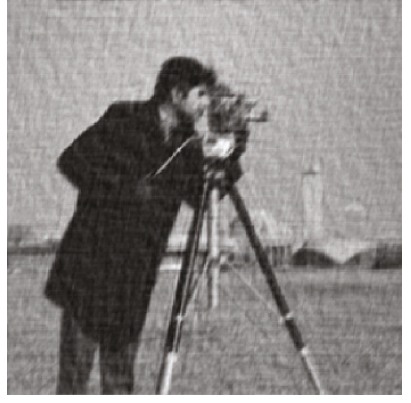

(b)

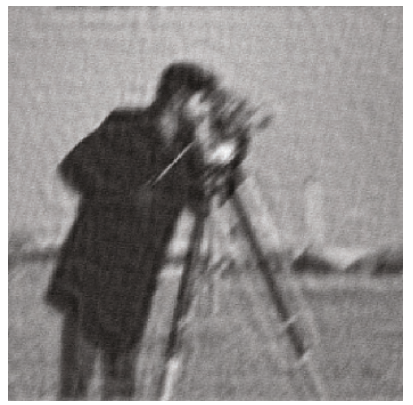

(d)

Figure 7: From Figure 6, we take a specific pair of values (BSNR, $\left.\varepsilon_{\sigma}\right)=(20 \mathrm{~dB}, 80 \%)$ showing the degraded image $\mathbf{y}$ in (a) and the restored images $\hat{\mathbf{x}}$ in (b), (c), and (d) when, respectively, obtained by the Wiener filter with optimum estimates (ISNR $=4.14 \mathrm{~dB}$ ), the same when an error of $\varepsilon_{\sigma}$ is applied on the noise variance (ISNR $=-3.25 \mathrm{~dB}$ ) and the last one when our proposed desensitisation method is used with the same error $($ ISNR $=1.44 \mathrm{~dB})$.

Logically, the ISNR value related to the Wiener filter with optimum estimates is always over those distributions. Let us remind that the error caused by the original image estimation, namely, $\left.S_{x x}\right|_{e} \cong S_{y y}$, is included into the parameter $C_{e}$ as well. Consequently, both methods yield an ISNR lower than the optimum one when $\varepsilon_{\sigma}=0$.

In order to present imaging results, let us take a specific pair of values (BSNR, $\left.\varepsilon_{\sigma}\right)$, that is, $(20 \mathrm{~dB}, 80 \%)$. Hence, we show the degraded image $\mathbf{y}$ in Figure $7(\mathrm{a})$ and the restored images $\hat{\mathbf{x}}$ in Figures $7(\mathrm{~b}), 7(\mathrm{c})$, and $7(\mathrm{~d})$ when respectively obtained by the Wiener filter with optimum estimates, the same when an estimation error of $\varepsilon_{\sigma}$ is applied on the noise variance and the last one when our proposed desensitisation method is used with the same error.

In full view of theses illustrations, we can ensure the benefits achieved by our method when errors on the noise variance are made. Certainly, an incremented noising effect is a consequence of the mistaken estimation $\varepsilon_{\sigma}$ as observed in the restored image of the Wiener approach in Figure $7(\mathrm{c})$. Yet, the desensitisation process is capable to nearly remove this artefact making the restored image Figure $7(\mathrm{~d})$ more approximate to the optimum one of Figure $7(\mathrm{~b})$ as stated by the ISNR, that is, a reached value of $1.44 \mathrm{~dB}$ from our restoration method improves the result of $-3.25 \mathrm{~dB}$ derived from the Wiener filter with wrong noise estimation and comes closer to the optimal of $4.14 \mathrm{~dB}$.
Going a step further, we can illustrate the associated function $Z(k)$ and detect the frequency pairs $\left(\omega_{i}, \omega_{j}\right)$ where desensitisation is reached, that is to say, $Z(k)<1$ as stated in (14). Figure 8 shows a binary image where desensitised frequencies are white coloured and the remainder of the spectrum appears black coloured. Looking at these illustrations, we can conclude that the desensitised frequencies are related to those eliminated by the lowpass degradation filter (i.e., to say, zeros which become poles in the restoration filter). Therefore, it means that the restoration process provides a sensitivity reduction where it is more likely to have magnified noise effects and, consequently, accomplishes better results than those obtained directly by the Wiener approach.

Example 2. In a second set of simulations, we deal with the case where a wrong estimation of the parameter $H_{e}$ is considered and only the fixed error related to the original spectral density $\left.S_{x x}\right|_{e} \cong S_{y y}$ has an effect on the parameter $C_{e}$, since the Gaussian noise is properly estimated by the real variance.

As well as Example 1, the original image is degraded by a motion blur using the same values, that is, 15 pixels and 45 degrees, and a Gaussian noise is added according to a definite BSNR of $20 \mathrm{~dB}$. Nonetheless, in the restoration process, the parameter $H_{e}$ is deviated from its real value by adjusting both of its descriptive factors, namely, the number of moved pixels $l$ and the inclination of the motion $\theta$. As previously 


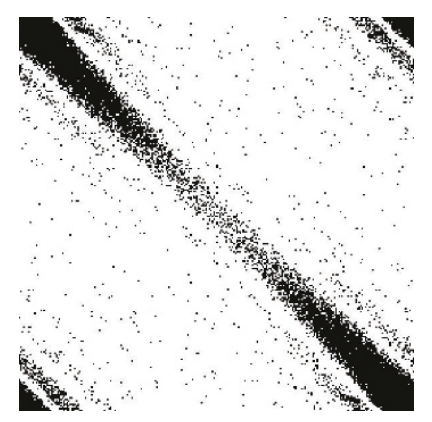

FIGURE 8: White coloured desensitised frequencies.

mentioned, the divergence of these parameters is expressed by means of the relative errors $\varepsilon_{l}$ and $\varepsilon_{\theta}$, respectively, whose definitions based on (35) as follow:

$$
\begin{gathered}
\varepsilon_{l}=\frac{l_{\text {real }}-l_{\text {estimated }}}{l_{\text {real }}} \cdot 100, \\
\varepsilon_{\theta}=\frac{\theta_{\text {real }}-\theta_{\text {estimated }}}{\theta_{\text {real }}} \cdot 100 .
\end{gathered}
$$

Similarly to (38), we express the estimates of those parameters as

$$
\begin{aligned}
& l_{\text {estimated }}=l_{\text {real }}\left(1-\frac{\varepsilon_{l}}{100}\right), \\
& \theta_{\text {estimated }}=\theta_{\text {real }}\left(1-\frac{\varepsilon_{\theta}}{100}\right) .
\end{aligned}
$$

Keeping the same guidelines as Example 1, we illustrate the distributions of ISNR obtained by both the Wiener filter $G$ and our desensitised restoration $G^{\prime}$, when the estimated parameters $l_{\text {estimated }}$ and $\theta_{\text {estimated }}$ are modified in relation to their respective errors. Regarding $\varepsilon_{l}$, we preserve the range between -100 and $100 \%$, but the value of $\varepsilon_{\theta}$ is wanted to make the angle vary within a sector of 180 degrees taking advantage of symmetry properties. Thus, it can be easily demonstrated that for an angle of 45 degrees, a range from -200 to $200 \%$ is required to fulfill that sector. Particularly, we can find these representations in Figures 9(a) and 9(b) addressed to show the influence of each parameter $l$ and $\theta$ on the results, always leaving one of them unalterable. Besides, a horizontal line is included symbolizing the constant value of ISNR reached when optimum estimates are considered in the Wiener filter.

Looking at those figures, we firstly draw a common conclusion regarding the target region, as previously defined as the range of errors where the value of ISNR obtained by the filter $G^{\prime}$ exceeds that of the Wiener approach $G$. On the whole, the desensitisation method achieves better results when considering high enough errors outside a relative narrow bandwidth located around low values of $\varepsilon_{l}$ and $\varepsilon_{\theta}$. Particularly, the distributions of ISNR for errors on the inclination $\theta_{\text {real }}$ follow an approximate symmetric shape, crossing in the values of angle from which successful results are goaled. On the other hand, estimates $l_{\text {estimated }}$ over the real value $l_{\text {real }}{ }^{\circ}$, namely, negative values of the error $\varepsilon_{l}$, obtain a significant enhancement thanks to desensitisation. Conversely, when reducing the number of pixels under $l_{\text {real }}{ }^{\circ}$, our restoration scheme yields quite similar values of ISNR to those reached by the Wiener filter.

Therefore, our proposed procedure is able to improve the quality of the restored image by the Wiener approach when making enough errors on whatever parameter of the degradation $H_{e}$. Furthermore, taking into account the benefits derived from Example 1 with respect to the estimation of noise, we give evidence to a corollary demonstrated in Section 3 (17), which stated that the global desensitisation of the filter $G$ is equally extended to whatever related parameter, for instance, $\sigma^{2}, l$, and $\theta$.

Nevertheless, the figures from both examples make obvious that our proposed restoration works better with errors on the noise variance than applying deviations from the degradation parameters as indicated by higher values of ISNR. Indeed, it can be extracted from the mathematical analysis in Section 3.4 where we can see that the improvements achieved for $\delta_{n}(k)$ were greater than the impairments obtained from $\delta_{r}(k)$, that is to say, a better behaviour with regards to noise.

Example 3. Finally, let us tackle an extreme problem where the estimates are not only modified regarding specific parameters, but also the noise and the PSF to be estimated as belonging to different classes from the original ones. Purposely, let us disturb the original image with a speckle noise and a "salt and pepper" artefact (we refer to two different kinds of noises) when a Gaussian estimation is considered. About PSF, a motion blur is estimated when the original degradation corresponds to responses such as the atmospheric turbulence phenomenon or the uniform blur.

On the subject of noise, we maintain a motion blur of 15 pixels and 45 degrees, but we apply a different noise having a variance $\sigma_{\text {real }}^{2}$ according to a BSNR of $10 \mathrm{~dB}$. In particular, a multiplicative noise is added by means of a uniformly distributed random noise with mean 0 and variance $\sigma_{\text {real }}^{2}$, namely, speckle noise. Conversely, a "salt and pepper" noise is added in proportion to a likelihood density of $2 \%$ making the resulted variance similar to $\sigma_{\text {real }}^{2}$. However, a Gaussian noise is once more estimated whose variance $\sigma_{\text {estimated }}^{2}$ is distorted by the relative error $\varepsilon_{\sigma}$ ranged between -100 and $100 \%$, keeping the parameter $H_{e}$ unalterable and leaving the fixed error related to the original spectral density $S_{x x} \cong S_{y y}$.

Following the same patterns of illustrations as the before analysed examples, let us draw the distributions of ISNR obtained by both the Wiener filter $G$ and our desensitised restoration $G^{\prime}$ when the estimated variance is modified in relation to $\varepsilon_{\sigma}$ for each input noise (Figures $10(\mathrm{a})$ and $10(\mathrm{~b})$ ). Paying attention to the target region, we reveal that our desensitised method achieves successful results regardless of the heterogeneity of noise estimates, as it can be obviously extracted from Figure 10(b) where our method always yields better values of ISNR than those from the Wiener approach for every error $\varepsilon_{\sigma}$. Although it is not so forceful with the speckle noise, there is always an enough value of the error $\varepsilon_{\sigma}$ from which the target region is reached. 


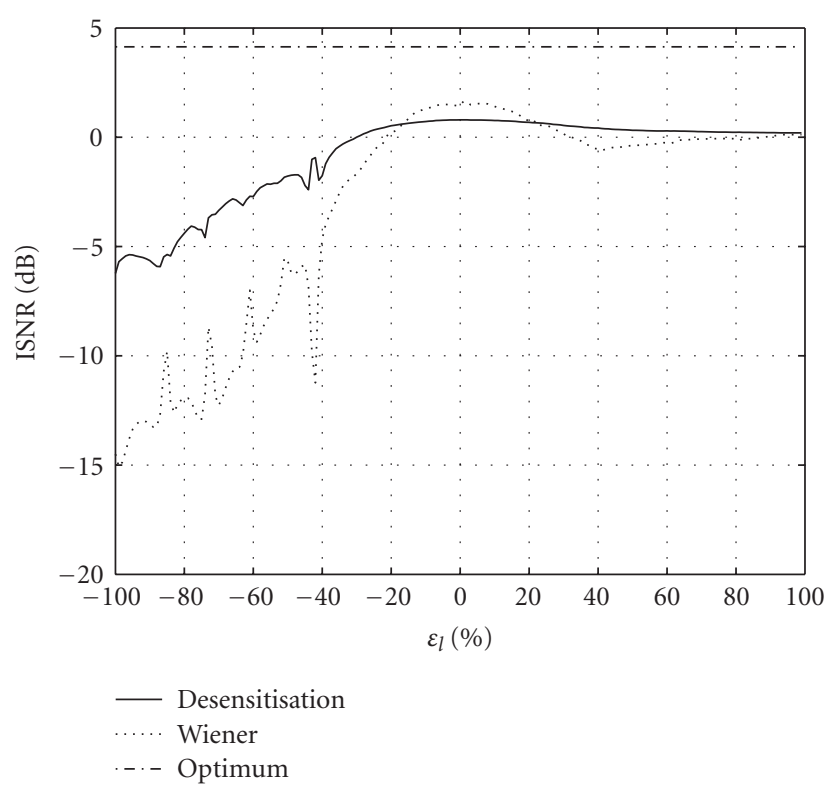

(a)

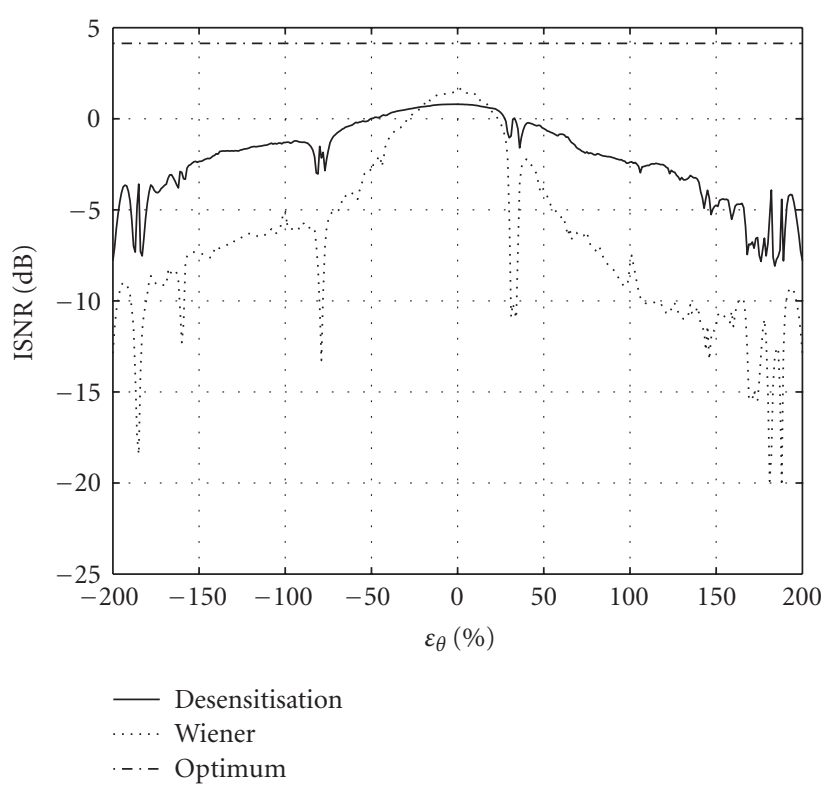

(b)

FIGURE 9: Distributions of ISNR obtained by both the Wiener filter and our desensitised method when the estimated parameters related to the PSF (motion blur) are altered, namely, the number of moved pixels (15 pixels) and the inclination (45 degrees) according to the errors $\varepsilon_{l}$ (a) and $\varepsilon_{\theta}$ (b), respectively. Regarding noise, a Gaussian artefact is applied in relation to a BSNR of $20 \mathrm{~dB}$ keeping the variance estimation unchanged. Besides, a horizontal line is included symbolizing the constant value of ISNR reached when optimum estimates are considered in the Wiener filter.

On the other hand, a Gaussian noise is added in relation to a variance indicated by a BSNR of $20 \mathrm{~dB}$ and the degradation is alternated between a rotationally symmetric Gaussian low-pass filter (turbulence atmospheric), specified by a square matrix of size $[15,15]$ and a standard deviation of 5 unities, and a circular averaging blur (uniform) within a square matrix of size $2 \cdot$ RADIUS +1 , where RADIUS $=5$. About estimates, we keep a Gaussian noise of the same variance, but we use a motion blur as PSF estimation whose inclination is established to 45 degrees and the length of pixels is changed around a value of 15 in accordance with a relative error $\varepsilon_{l}$.

From Figures 11(a) and 11(b), we come up to the same conclusion about the effectiveness of our desensitised scheme, getting better results over the Wiener approach even when a variation of the degradation estimation is considered. Let us point out that these figures follow the same patterns of the analogous Figure 9(a) and yet again keep worse values of ISNR compared to those with respect to noise estimation.

\section{EXTENDED RESULTS}

Hitherto, we have showed how our innovative method is able to desensitise the Wiener restoration filter regarding wrong estimates on the dependant parameters. However, our intention is now to extend the validity of our proposal by providing other fair comparisons with the state-of-art.
One of the issues to be addressed is the way we are considering wrong estimates of the parameters. As stated in (35), a relative error $\varepsilon_{P}$ allows the misspecification of a parameter knowing the real value. Yet, it is worthy to know the actual values reached when using real estimation methods and how our desensitisation scheme works consequently.

On the other hand, we aim to specify the restoration filter $G$ with another more sophisticated technique than the Wiener method, giving evidence of the functionality of the desensitisation in a general sense.

By keeping the same test image Cameraman, we cope with the before-mentioned tasks right through the next two sections.

\subsection{Real estimates}

In Section 1, we cited some bibliography with regards to the estimation of the PSF and now it comes the time to draw on those methods. Hence, the parameter $H_{e}$ is intended to be obtained by applying an estimation technique with no more information than the degraded image $y$. Conversely, going on the Wiener context, the other parameter $C_{e}$ is only deviated from its real value by the approximation $\left.S_{x x}\right|_{e} \cong S_{y y}$, since the input Gaussian noise is properly estimated by the real variance.

Firstly, let us start with a rough estimation of the PSF, as provided by the Radon transform [13], performed on a binary representation of the spectrum $Y$ when using an 


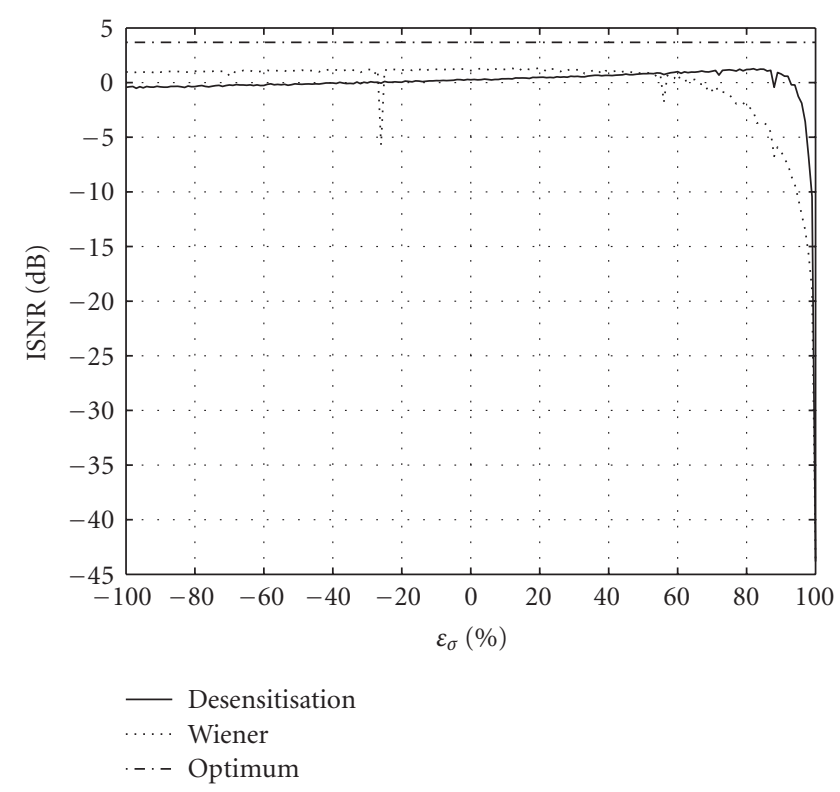

(a)

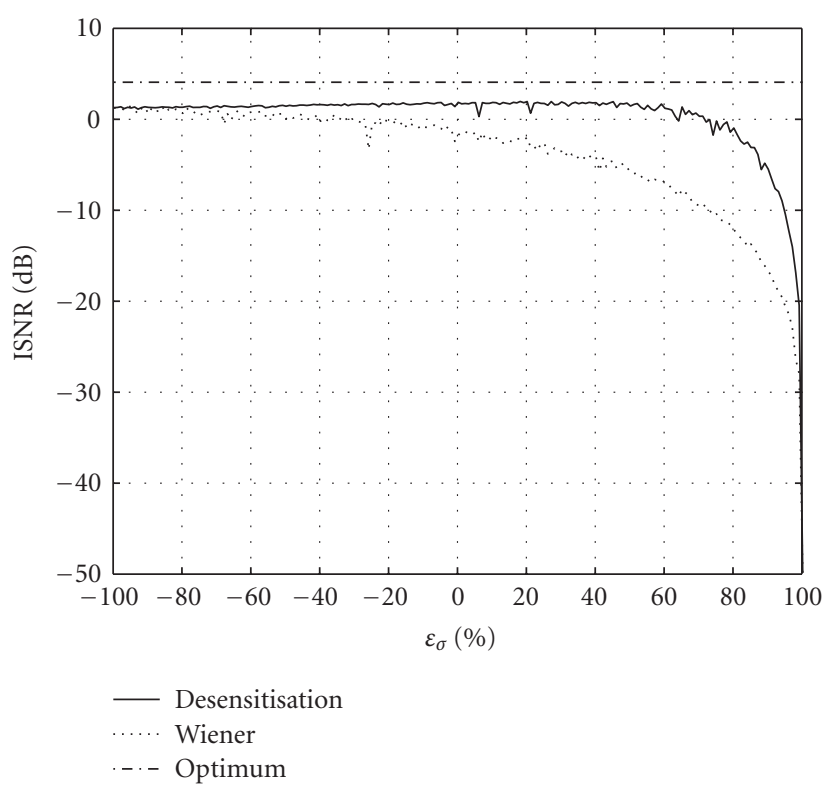

(b)

FIGURE 10: Distributions of ISNR obtained by both the Wiener filter and our desensitised method when a Gaussian noise is estimated according to a BSNR of $10 \mathrm{~dB}$ whose variance is altered by $\varepsilon_{\sigma}$ while a multiplicative noise (a) and a "salt and pepper" artefact (b) are added to the original image. Regarding PSF, the estimated parameter keeps unchanged. Besides, a horizontal line is included symbolizing the constant value of ISNR reached when optimum estimates are considered in the Wiener filter.

appropriate threshold to enhance the nearer zero frequencies. Expressly, we degrade the original image according to a motion blur described by a length of 15 pixels and an angle of 45 degrees in a counter-clockwise direction. Later on, a Gaussian noise is added following a variable BSNR. Thus, we yield a thresholded spectrum of the degraded image in circumstances without noise as illustrated in Figure 12.

Having a look to this figure, we observe how the zeros of the degradation filter are highlighted, making possible to calculate the inclination of the motion by means of the Radon transform. Indeed, our example deals with the estimation of the PSF, assuming a motion blur with the real length of 15 pixels, but computing the parameter $\theta_{\text {estimated }}$ in a wide range of noise ratios. Afterwards, the estimated value of $H_{e}$ is used within a direct Wiener restoration compared to the results achieved by our desensitisation model.

In the light of the above, we build Table 2 collecting the respective values of ISNR for every $\theta_{\text {estimated. }}$. It is obviously noted that this estimation gets worse as the noise intensity increases, even acquiring a random behaviour. Consequently, the Wiener restoration outcomes continuously poorer results which are improved by our proposed method when the value of $\theta_{\text {estimated }}$ is sufficiently deviated from its desirable 45 degrees. So, we come up to the same conclusion indicated in Example 2, where the target region, namely, the range of errors where the value of ISNR obtained by the filter $G^{\prime}$ exceeds that of the Wiener approach $G$, is reached within a wide range of high enough errors.
Therefore, our desensitisation scheme becomes a way to counteract the weakness of the Radon method with respect to the noise.

Next, let us tackle another more complex estimation technique based on the eigenvalues of the degraded image $\mathbf{y}$ [14] which requires its singular value decomposition as

$$
y=U_{y} S_{y} V_{y}^{T},
$$

where $U_{y}$ and $V_{y}$ are orthonormal matrices which satisfy the conditions $U_{y} U_{y}^{T}=I$ and $V_{y} V_{y}^{T}=I$ with $I$ as the identity matrix, and $S_{y}$ is the matrix of eigenvalues which are related to each one of the rows from the matrices $U_{y}$ and $V_{y}$ (eigenvectors).

As stated in the bibliography, the quality of the PSF estimation depends on the number of eigenvectors $R$ (with their respective eigenvalues) to be considered, since the minor eigenvalues have less influence on the definition of the image and, furthermore, they may hold noise artefacts.

By means of this estimation method, we obtain entirely the structure of the PSF matrix. Thus, let us provide a parameter to measure how close the estimation is to the real matrix:

$$
\Delta h=\frac{\sum_{i=1}^{A} \sum_{j=1}^{B}\left|h_{e}(i, j)-h(i, j)\right|}{A \cdot B},
$$

where $h(i, j)$ and $h_{e}(i, j)$ are the respective real and estimated $A \times B$ sized PSFs. 


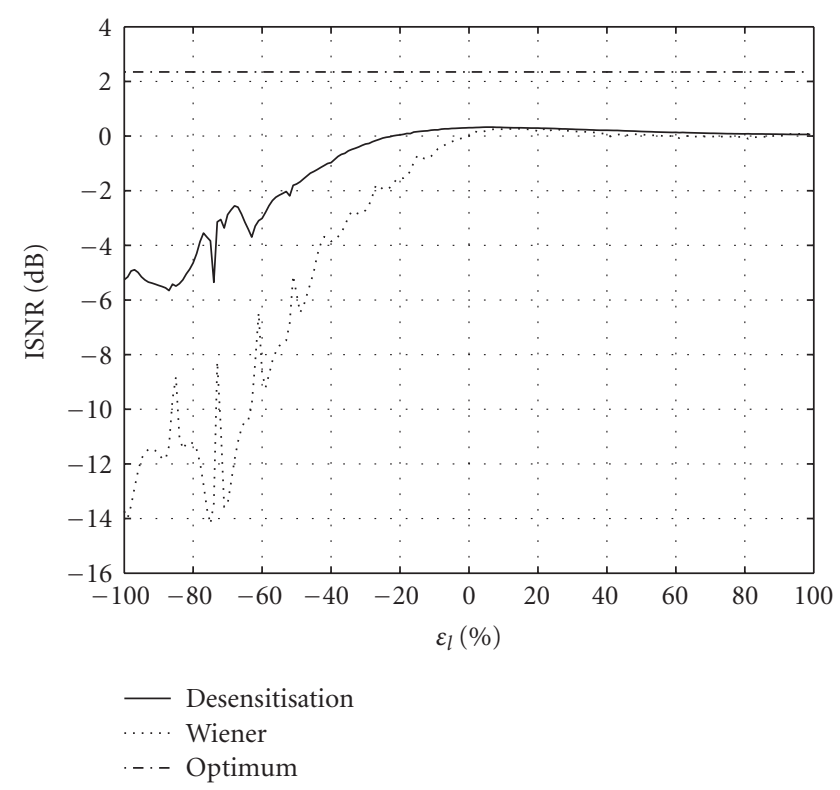

(a)

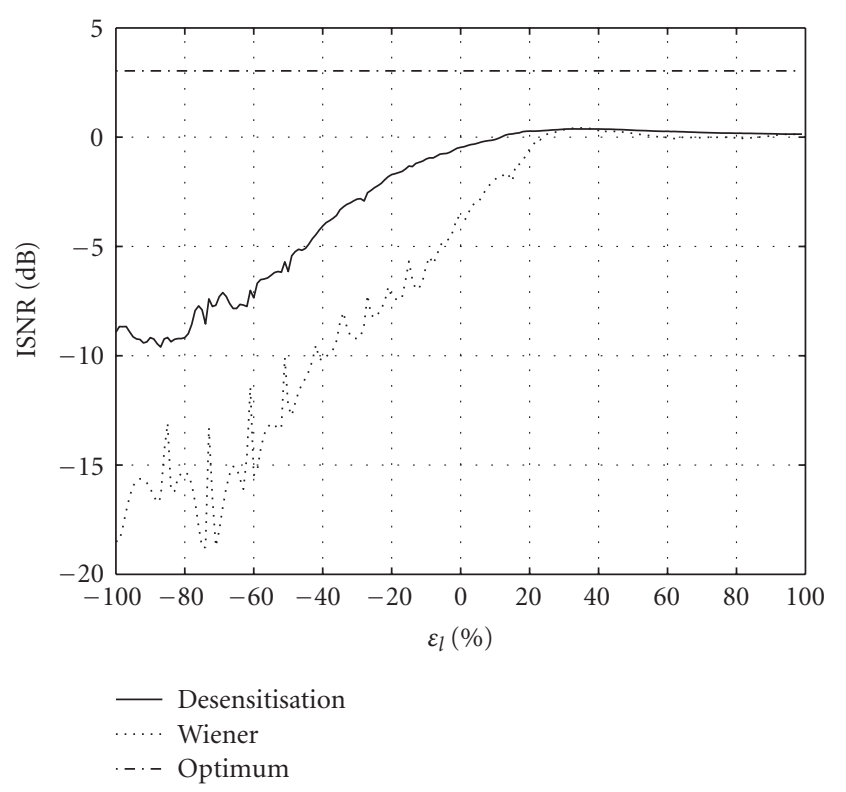

(b)

FIGURE 11: Distributions of ISNR obtained by both the Wiener filter and our desensitised method when a motion blur is estimated according to an inclination of 45 degrees and a number of 15 pixels altered by $\varepsilon_{l}$ while a Gaussian low-pass filter (a) and a uniform blur (b) are applied on the original image. Regarding noise, a Gaussian artefact is considered in relation to a BSNR of $20 \mathrm{~dB}$. Besides, a horizontal line is included symbolizing the constant value of ISNR reached when optimum estimates are considered in the Wiener filter.

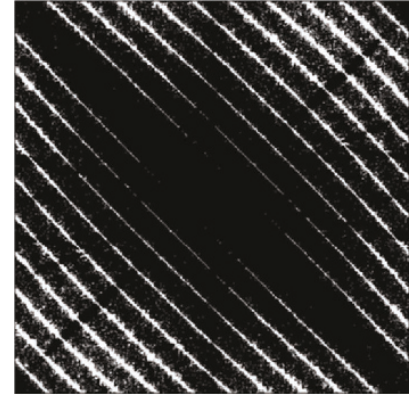

FIGURE 12: Spectrum of the motion blurred image applying a threshold.

Once all the required elements are defined, we carry out some specific samples. In particular, the original image is degraded by a $3 \times 3$ Gaussian blur with a variable standard deviation and an additive Gaussian blur according to a BSNR relation of $40 \mathrm{~dB}$, that is to say, low noise conditions in order to prove exclusively the influence of the standard deviation. Then, we aim to estimate the PSF matrix by applying the eigenvalues method and computing so both Wiener and desensitised restorations.

Looking at Table 3, we find the ISNR results when ranging the standard deviation from 1 to 5 and taking a number of 10 or 20 eigenvectors, apart from the respective errors on the PSF estimation. Yet again, we draw the same conclusion kept all along the paper, explicitly, better results with the desensitisation method when having high enough errors on the estimation, although, in this case, it is only necessary a low error to reach significant improvements.

Furthermore, it means that our proposed scheme becomes a strong way to counteract the variation of the standard deviation on the eigenvalues method. Therefore, the desensitised restoration takes a place to solve the weakness of some estimation methods concerning specific parameters.

\subsection{SAR restoration}

Going a step further in our objective to achieve fair comparisons with the state-of-art, the generic restoration filter $G$ is now substituted by the well-known Bayesian algorithm when using a simultaneous autoregressive (SAR) prior distribution $[37,38]$.

From these references, it is taken out that the said restoration is based on a constrained minimisation process as follows:

$$
\widehat{x}=\min _{\hat{x}}\left\{\alpha\|C \hat{\mathbf{x}}\|^{2}+\beta\|\mathbf{y}-\mathbf{H} \hat{\mathbf{x}}\|^{2}\right\}
$$

written in a lexicographic manner, where $C$ is the Laplacian operator, $\alpha$ is a parameter equivalent to the inverse of the prior variance which controls the smoothness of the restoration, and $\beta$ is the inverse of the noise variance. 
TABLE 2: Set of ISNR results obtained by both the Wiener filter and our proposed method when using estimates of a motion PSF (length of 15 pixels and inclination of 45 degrees) computed by the Radon transform in different Gaussian noise conditions (the noise variance is estimated with the actual value). The respective estimated values of $\theta_{\text {estimated }}$ are showed, leaving the $l_{\text {estimated }}$ to the real value.

\begin{tabular}{c|c|rrrrrrr}
\hline \multicolumn{2}{c}{} & \multicolumn{5}{c}{ BSNR $(\mathrm{dB})$} \\
\cline { 4 - 8 } \multicolumn{2}{c|}{} & \multicolumn{2}{c|}{$\theta_{\text {estimated }}$ (degrees) } & 40 & 35 & 30 & 25 & 20 \\
\hline \multirow{3}{*}{ ISNR (dB) } & Optimum estimates & Wiener & 10.29 & 8.40 & 6.73 & 5.28 & 4.14 & 3.48 \\
\cline { 2 - 8 } & \multirow{2}{*}{ Radon estimates } & Wiener & 4.64 & 2.27 & -1.25 & -2.64 & -0.81 & 0.03 \\
& & Desensitisation & 2.50 & 1.65 & 0.70 & -0.21 & 0.36 & 0.76 \\
\hline
\end{tabular}

TABLE 3: Set of ISNR results obtained by both the Wiener filter and our proposed method when using estimates of a Gaussian $3 \times 3$ sized PSF computed by the algorithm of eigenvalues with different values for the standard deviation and specified to 10 and 20 eigenvectors $R$. Low-noise intensity is considered by a ratio of BSNR to $40 \mathrm{~dB}$, keeping the estimated noise variance to the real value. The respective values of error $\Delta h$ per estimation of PSF are indicated.

\begin{tabular}{|c|c|c|c|c|c|c|c|c|c|c|c|}
\hline \multirow{2}{*}{\multicolumn{3}{|c|}{$R=10$}} & \multicolumn{9}{|c|}{$\sigma$} \\
\hline & & & 1 & 1.5 & 2 & 2.5 & 3 & 3.5 & 4 & 4.5 & 5 \\
\hline \multicolumn{3}{|c|}{$\Delta h$} & 0.0019 & 0.0207 & 0.0279 & 0.0312 & 0.0330 & 0.0341 & 0.0348 & 0.0353 & 0.0357 \\
\hline \multirow{3}{*}{ ISNR (dB) } & Optimum estimates & Wiener & 6.86 & 8.06 & 8.33 & 8.45 & 8.57 & 8.60 & 8.60 & 8.60 & 8.62 \\
\hline & \multirow{2}{*}{ Eigenvalues estimates } & Wiener & 3.37 & 2.25 & 0.86 & 0.06 & -0.44 & -0.77 & -1.00 & -1.16 & -1.28 \\
\hline & & Desensitisation & 1.49 & 1.52 & 1.34 & 1.21 & 1.12 & 1.05 & 1.00 & 0.97 & 0.94 \\
\hline \multirow{2}{*}{\multicolumn{3}{|c|}{$R=20$}} & \multicolumn{9}{|c|}{$\sigma$} \\
\hline & & & 1 & 1.5 & 2 & 2.5 & 3 & 3.5 & 4 & 4.5 & 5 \\
\hline \multicolumn{3}{|c|}{$\Delta h$} & 0.0052 & 0.0237 & 0.0309 & 0.0343 & 0.0361 & 0.0372 & 0.0380 & 0.0385 & 0.0388 \\
\hline \multirow{3}{*}{ ISNR (dB) } & Optimum estimates & Wiener & 6.86 & 8.06 & 8.33 & 8.45 & 8.57 & 8.60 & 8.60 & 8.60 & 8.62 \\
\hline & \multirow{2}{*}{ Eigenvalues estimates } & Wiener & 3.27 & 1.11 & -0.85 & -2.45 & -3.74 & -4.72 & -5.46 & -5.99 & -6.37 \\
\hline & & Desensitisation & 1.48 & 1.34 & 1.07 & 1.07 & 1.17 & 1.22 & 1.18 & 1.14 & 1.10 \\
\hline
\end{tabular}

Following an ordinary minimisation, as detailed in [2], it leads to

$$
\hat{x}=\left(\mathbf{H}^{T} \mathbf{H}+\lambda \mathbf{C}^{T} \mathbf{C}\right)^{-1} \mathbf{H}^{T} \mathbf{y},
$$

where $\lambda$ is the so-called Lagrange multiplier defined as

$$
\lambda=\frac{\alpha}{\beta}
$$

whose value is estimated by means of the Bayesian paradigm [37, 38].

Presuming a linear and spatial-invariant degradation and considering images as periodic matrices, we may diagonalise the terms in (45) with the purpose of getting the representation in the Fourier transform

$$
X\left(\omega_{i}, \omega_{j}\right)=\frac{H^{*}\left(\omega_{i}, \omega_{j}\right)}{\left|H^{*}\left(\omega_{i}, \omega_{j}\right)\right|^{2}+\lambda\left|P\left(\omega_{i}, \omega_{j}\right)\right|^{2}} Y\left(\omega_{i}, \omega_{j}\right)
$$

and, therefore, we achieve the definition of the restoration filter

$$
G\left(\omega_{i}, \omega_{j}\right)=\frac{H^{*}\left(\omega_{i}, \omega_{j}\right)}{\left|H^{*}\left(\omega_{i}, \omega_{j}\right)\right|^{2}+\lambda\left|P\left(\omega_{i}, \omega_{j}\right)\right|^{2}},
$$

where $P\left(\omega_{i}, \omega_{j}\right)$ stands for the DFT of the Laplacian operator.
The next step consists of extending the concept of desensitisation on this restoration filter, though analytically it remains the same conclusions since it was previously managed in a general sense $G$. Then, let us rearrange Examples 1 and 2 from last section keeping the same motion blur of 15 pixels and 45 degrees, but adding a Gaussian noise of a specific ratio of $\mathrm{BSNR}=20 \mathrm{~dB}$.

Regarding the estimation of the noise, the relative error $\mathcal{E}_{\sigma}$ affects directly the Lagrange parameter $\lambda$ and, thus, making results relatively far from the desired optimum according to (44). However, looking at Figure 13, we demonstrate once more that the desensitised filter $G^{\prime}$ is able to improve the ISNR results with respect to those obtained by the original restoration $G(\mathrm{SAR})$. Specifically, the so-mentioned target area starts from a reasonably small error, under $20 \%$, and reaches a quite pronounced comparative quality, even achieving results close to the optimum during a significant range of errors. Nonetheless, it is only verified at the positive side of the $\varepsilon_{\sigma}$, that is to say, $\sigma_{\text {estimated }}^{2}<\sigma_{\text {real }}^{2}$ as inferred from (39), but this behaviour may be expanded if considering other values of the ratio BSNR.

In other words, our desensitisation scheme becomes a robust way to compensate the miscalculation of the parameter $\lambda$ by the Bayesian algorithm when having unreal values of the noise variance. Let us make a reference to the constant 


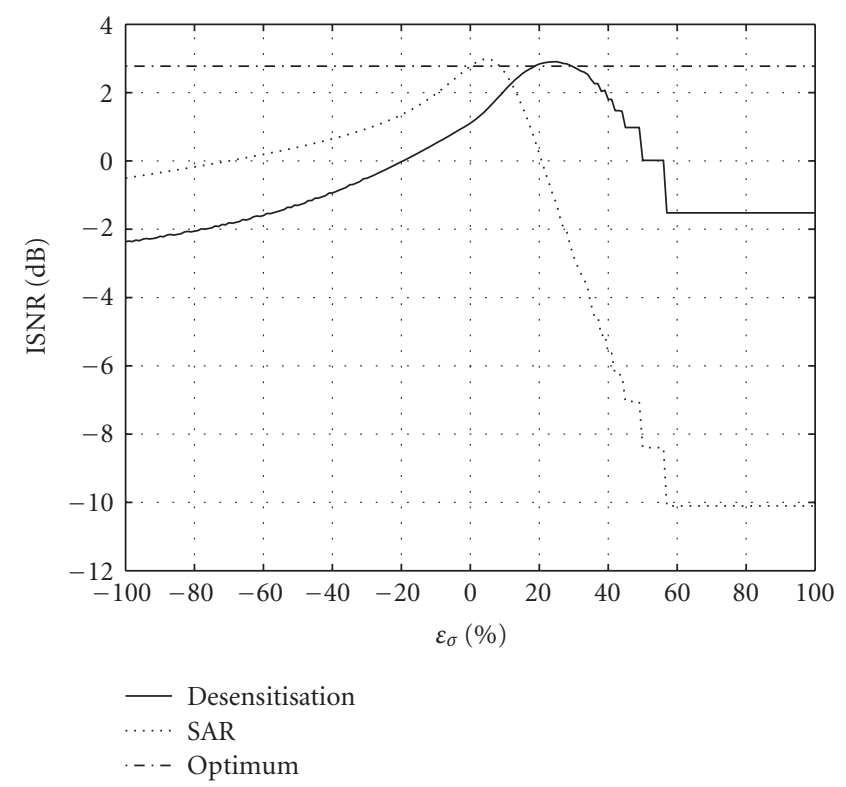

FIGURE 13: Distributions of ISNR obtained by both the SAR restoration and our desensitised method when the estimated Gaussian noise variance $(\mathrm{BSNR}=20 \mathrm{~dB}$ ) is altered according to a relative error $\varepsilon_{\sigma}$ leaving the PSF estimation unchanged (motion blur). Besides, a horizontal line is included symbolizing the constant value of ISNR reached when optimum estimates are considered in the SAR filter.

regions of the last illustration with the intention of making obvious a saturated state of the Bayesian procedure for some noise conditions.

Finally, we accomplish the same simulation of Example 2 within the SAR context, but only choosing the variation of the parameter $\theta$, namely, the inclination of the motion blur. Another comparative representation of the ISNR diagrams (Figure 14) keeps what is definitely a contrasted conclusion about the improvement of our desensitisation process whether having errors superior to a concrete range.

Thought should be given to the fact that the estimation error of the PSF causes an overall impairment on the SAR restoration (47), in both $H$ and $\lambda$ parameters, and the desensitisation is then in charge of counteracting the effects on the results.

As a matter of interest, a deeper analysis of how the Lagrange multiplier is influenced by the desensitisation could open up new future researches.

\section{CONCLUSIONS}

In this paper, we presented an iterative algorithm within the Fourier transform domain addressed to reduce the sensitivity of a general restoration filter (but specified to the Wiener approach) with respect to the dependant parameters.

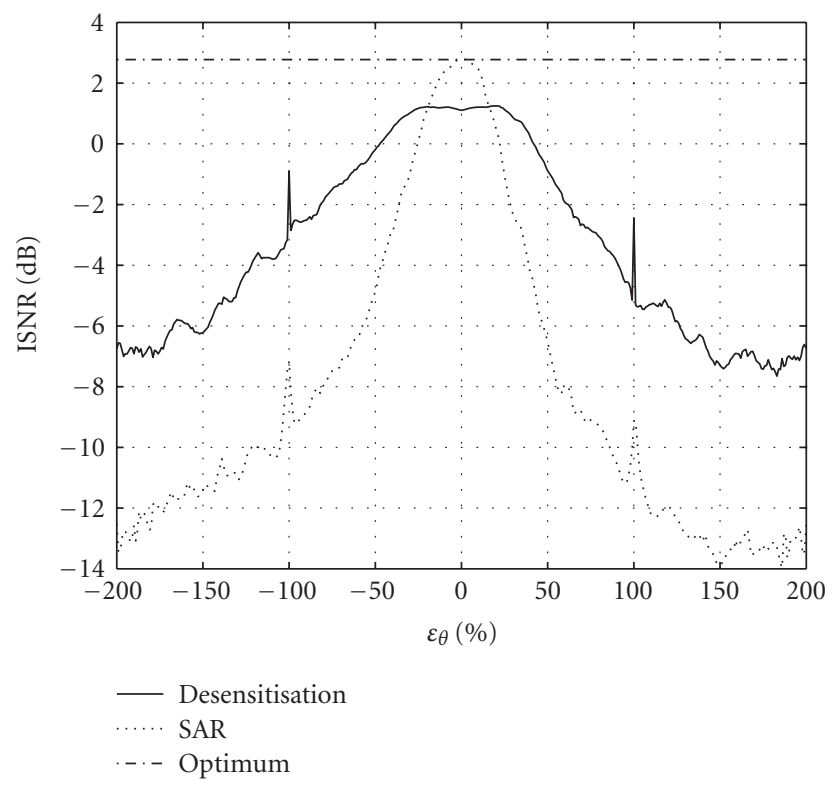

FIGURE 14: Distributions of ISNR obtained by both the SAR restoration and our desensitised method when the inclination of the motion PSF is altered from 45 degrees according to the error $\varepsilon_{\theta}$, keeping the estimated length parameter to the actual value of 15 pixels. Regarding noise, a Gaussian artefact is applied in relation to a BSNR of $20 \mathrm{~dB}$ keeping the variance estimation unchanged. Besides, a horizontal line is included symbolizing the constant value of ISNR reached when optimum estimates are considered in the Wiener filter.

A highly detailed analysis showed the requirement of a trade-off to reach a number of iterations applied to each frequency component, in order to balance the desensitisation degree and the restoration error level.

First results of the desensitisation influence on both the image-dependent and noise-dependent errors revealed a higher robustness on the noise estimation as stated later in the examples.

In case of errors on the variance of the noise, we justified how our proposed method achieves ISNR values better than those obtained when using the same wrong estimates in the Wiener approach. Expressly, it is more likely to reach the target region when having high-noise conditions. Furthermore, we could demonstrate that the desensitised frequency pairs $\left(\omega_{i}, \omega_{j}\right)$ are related to those eliminated by the low-pass degradation filter, that is to say, zeros which become poles in the restoration filter and, therefore, preventing from magnified noise effects.

On the other hand, deviations from the original parameters regarding the PSF were equally analysed making obvious the successful behaviour of the filter regardless of whatsoever estimation to be disturbed.

Finally, a set of extended results aimed to achieve fair comparisons of our proposed method with regards to the state-of-art. Indeed, real estimation methods and another original restoration (SAR) were applied with successful results. 


\section{APPENDICES}

\section{A. DERIVATION OF (31)}

By considering the definition of $Z(k)$ from (13) and applying its first differentiation with respect to $k$, we have

$$
\begin{aligned}
R(k) & =\frac{\partial}{\partial k}\left\{\frac{\partial}{\partial k}\left[(k+1)\left(G H_{e}\right)^{k}\right]\right\} \\
& =\frac{\partial}{\partial k}\left\{\left(G H_{e}\right)^{k}+(k+1)\left(G H_{e}\right)^{k} \ln \left(G H_{e}\right)\right\} \\
& =\frac{\partial}{\partial k}\left\{\left(G H_{e}\right)^{k}\left[1+(k+1) \ln \left(G H_{e}\right)\right]\right\} .
\end{aligned}
$$

Taking a new derivative, it leads to

$$
\begin{aligned}
R(k)= & \left(G H_{e}\right)^{k} \ln \left(G H_{e}\right)\left[1+(k+1) \ln \left(G H_{e}\right)\right] \\
& +\left(G H_{e}\right)^{k} \ln \left(G H_{e}\right) .
\end{aligned}
$$

Thus, we can simplify to

$$
R(k)=\left(G H_{e}\right)^{k} \ln \left(G H_{e}\right)\left[2+(k+1) \ln \left(G H_{e}\right)\right] .
$$

\section{B. DERIVATION OF (33)}

We start by solving the optimisation problem which states the calculation of a value of $k$ which maximises the function $R(k)$. Hence, let us compute its first derivative with respect to $k$ and set it to zero. Then, from (31), we obtain

$$
\begin{aligned}
\frac{\partial R(k)}{\partial k} & =\frac{\partial}{\partial k}\left\{\left(G H_{e}\right)^{k} \ln \left(G H_{e}\right)\left[2+(k+1) \ln \left(G H_{e}\right)\right]\right\} \\
& =\left(G H_{e}\right)^{k} \ln ^{2}\left(G H_{e}\right)\left[3+(k+1) \ln \left(G H_{e}\right)\right]=0 .
\end{aligned}
$$

Considering the regularisation range from (20), the case of $G H_{e}=0$ is a potential solution from $\left(G H_{e}\right)^{k}$, but it is undetermined for the neperian function. Consequently, we can solve (B.1) to find the position of the maximum of $R(k)$ for whatever value $0<G H_{e}<1$ as

$$
3+(k+1) \ln \left(G H_{e}\right)=0
$$

Therefore,

$$
K=\operatorname{round}\left\{-\left[1+\frac{3}{\ln \left(G H_{e}\right)}\right]\right\},
$$

where round $\{\cdot\}$ represents the operator to turn $K$ into an integer value such that $k \geq 1$.

Let us check now that $R(k)$ shows actually a maximum for this value $K$ given by (B.3). Applying the second derivative of $R(k)$ from (B.1) and imposing the condition to be negative for $K$,

$$
\begin{aligned}
\left.\frac{\partial^{2} R(k)}{\partial k^{2}}\right|_{K} & =\left.\frac{\partial}{\partial k}\right|_{K}\left\{\left(G H_{e}\right)^{k} \ln ^{2}\left(G H_{e}\right)\left[3+(k+1) \ln \left(G H_{e}\right)\right]\right\} \\
& =\left.\left(G H_{e}\right)^{k} \ln ^{3}\left(G H_{e}\right)\left[4+(k+1) \ln \left(G H_{e}\right)\right]\right|_{K}<0 .
\end{aligned}
$$

Taking the before-mentioned range $0<G H_{e}<1$ into account, we can assure that there will be a maximum in $R(k)$ whether,

$$
4+\left.(k+1) \ln \left(G H_{e}\right)\right|_{K}>0 .
$$

Substituting (B.3) into (B.5), we can simplify to a first constraint on $G H_{e}$,

$$
G H_{e}>\exp (-2) \approx 0.14 \text {. }
$$

On the other hand, let us ensure now that this total number of iterations $K$ recommended by (B.3) also satisfies the condition established for the filter $G^{\prime}$ to be less sensitive than $G$ defined as $Z(k)<1$ in expression (14). Thus, substituting the value of $k$ from (B.3) into the function $Z(k)(13)$ and imposing the latter condition, we have the following equation to be solved:

$$
\left.Z(k)\right|_{K}=\left(G H_{e}\right)^{-\left[1+3 / \ln \left(G H_{e}\right)\right]}\left[\frac{3}{\ln \left(G H_{e}\right)}\right]<1 .
$$

On the basis of [39], we achieve the solution regarding $G H_{e}$ as

$$
G H_{e}<\frac{-3}{\exp (-3) \text { LambertW }(-3 / \exp (-3))} \approx 0.84,
$$

where the operation LambertW $\{\cdot\}$ can be defined as

$$
\operatorname{LambertW}(x) \cdot \exp [\operatorname{LambertW}(x)]=x .
$$

Therefore, the number of iterations $K$ aimed at (B.3) fulfills the conditions from (34) if and only if

$$
0.14<G H_{e}<0.84 \text {. }
$$

\section{REFERENCES}

[1] H. C. Andrews and B. R. Hunt, Digital Image Restoration, Prentice-Hall, Englewood Cliffs, NJ, USA, 1977.

[2] R. C. Gonzalez and P. Wintz, Digital Image Processing, Addison Wesley, Reading, Mass, USA, 1992.

[3] N. Tikhonov and V. Y. Arsenin, Solutions of Ill-Posed Problems, Scripta Series in Mathematics, John Wiley \& Sons, New York, NY, USA, 1977. 
[4] M. R. Banham and A. K. Katsaggelos, "Digital image restoration,” IEEE Signal Processing Magazine, vol. 14, no. 2, pp. 2441, 1997.

[5] R. L. Lagendijk, A. M. Tekalp, and J. M. Biemond, "Maximum likelihood image and blur identification: a unifying approach," Optical Engineering, vol. 29, no. 5, pp. 422-435, 1990.

[6] S. J. Reeves and R. M. Mersereau, "Blur identification by the method of generalized cross-validation," IEEE Transactions on Image Processing, vol. 1, no. 3, pp. 301-311, 1992.

[7] A. M. Tekalp, H. Kaufman, and J. W. Woods, "Identification of image and blur parameters for the restoration of noncausal blurs," IEEE Transactions on Acoustics, Speech, and Signal Processing, vol. 34, no. 4, pp. 963-972, 1986.

[8] Y.-L. You and M. Kaveh, "A regularization approach to joint blur identification and image restoration," IEEE Transactions on Image Processing, vol. 5, no. 3, pp. 416-428, 1996.

[9] G. B. Giannakis and R. W. Heath Jr., "Blind identification of multichannel FIR blurs and perfect image restoration," IEEE Transactions on Image Processing, vol. 9, no. 11, pp. 1877-1896, 2000.

[10] C. Feng, J.-W. Ma, and J.-P. Chen, "The PSF correction method for satellite image restoration," in Proceedings of the 3rd International Conference on Image and Graphics, pp. 3134, Hong Kong, December 2004.

[11] M. Ciftci and D. B. Williams, "Optimal estimation and sequential channel equalization algorithms for chaotic communications systems," EURASIP Journal on Applied Signal Processing, vol. 2001, no. 4, pp. 249-256, 2001.

[12] L. Galleani, L. Cohen, D. Nelson, and J. D. Scargle, "Instantaneous spectrum estimation from event-based densities," EURASIP Journal on Applied Signal Processing, vol. 2002, no. 1, pp. 87-91, 2002.

[13] M. Hayenga, S. Swan, and A. Zaharias, "Point Spread Function Estimation for the purpose of Motion Blurred Image Enhancement," December 2002.

[14] Z. Devcic and S. Loncaric, "Blur identification using averaged spectra of degraded image singular vectors," in Proceedings of IEEE International Conference on Acoustics, Speech, and Signal Processing (ICASSP '00), vol. 4, pp. 2195-2198, Istanbul, Turkey, June 2000.

[15] R. Molina, A. K. Katsaggelos, J. Mateos, A. Hermoso, and C. A. Segall, "Restoration of severely blurred high range images using stochastic and deterministic relaxation algorithms in compound Gauss-Markov random fields," Pattern Recognition, vol. 33, no. 4, pp. 555-571, 2000.

[16] N. Balram and J. M. F. Moura, "Noncausal Gauss-Markov random fields: parameter structure and estimation," IEEE Transactions on Information Theory, vol. 39, no. 4, pp. 1333-1355, 1993.

[17] F. C. Jeng and J. W. Woods, "Compound Gauss-Markov models for image processing," in Digital Image Restoration, A. K. Katsaggelos, Ed., vol. 23 of Springer Series in Information Science, Springer, Berlin, Germany, 1991.

[18] M. Martin-Fernandez, R. San Josa-Estepar, C.-F. Westin, and C. Alberola-Lopez, "A novel Gauss-Markov random field approach for regularization of diffusion tensor maps," in 9th International Conference on Computer Aided Systems Theory (EUROCAST '03), vol. 2809 of Lecture Notes in Computer Science, pp. 506-517, Las Palmas de Gran Canaria, Spain, February 2003.

[19] J. K. Romberg, H. Choi, and R. G. Baraniuk, "Bayesian tree-structured image modeling using wavelet-domain hid- den Markov models," IEEE Transactions on Image Processing, vol. 10, no. 7, pp. 1056-1068, 2001.

[20] H. Choi and R. G. Baraniuk, "Wavelet statistical models and Besov spaces," in Wavelet Applications in Signal and Image Processing VII, vol. 3813 of Proceedings of SPIE, pp. 489-501, Denver, Colo, USA, July 1999.

[21] S. G. Mallat, "Multifrequency channel decompositions of images and wavelet models," IEEE Transactions on Acoustics, Speech, and Signal Processing, vol. 37, no. 12, pp. 2091-2110, 1989.

[22] V. Koivunen, "A robust nonlinear filter for image restoration," IEEE Transactions on Image Processing, vol. 4, no. 5, pp. 569578, 1995.

[23] S. Voloshynovskiy, "Robust image restoration based on concept of M-estimation and parametric model of image spectrum," in Proceedings of the 5th International Workshop on Systems, Signals and Image Processing (IWSSIP '98), pp. 123-126, Zagreb, Croatia, June 1998.

[24] H. Allende, J. Galbiati, and R. Vallejos, "Digital image restoration using autoregressive time series type models," in Anais IX Simp'sio Brasileiro de Sensoriamento Remoto, pp. 1017-1027, Santos, Brasil, September 1998.

[25] A. D. Hillery and R. T. Chin, "Iterative Wiener filters for image restoration," IEEE Transactions on Signal Processing, vol. 39, no. 8, pp. 1892-1899, 1991.

[26] R. L. Lagendijk, J. Biemond, and D. E. Boekee, "Regularized iterative image restoration with ringing reduction," IEEE Transactions on Acoustics, Speech, and Signal Processing, vol. 36, no. 12, pp. 1874-1888, 1988.

[27] J. G. Nagy, R. J. Plemmons, and T. C. Torgersen, "Iterative image restoration using approximate inverse preconditioning," IEEE Transactions on Image Processing, vol. 5, no. 7, pp. 11511162, 1996.

[28] C. Erbas and S. Kent, "A new iterative technique for image restoration of ERS-2 raw data," in Proceedings of International Conference on Recent Advances in Space Technologies (RAST '03), pp. 85-90, Istanbul, Turkey, November 2003.

[29] M. Haind, "Recursive model-based image restoration," in Proceedings of the 15th International Conference on Pattern Recognition (ICPR '00), vol. 3, pp. 342-345, Barcelona, Spain, September 2000.

[30] S. J. Click and W. Xia, "Iterative restoration of SPECT projection images," IEEE Transactions on Nuclear Science, vol. 44, no. 2, pp. 204-211, 1997.

[31] J. I. Moon, S. K. Kim, J. K. Paik, and M. G. Kang, "Fast iterative image restoration algorithms," in Proceedings of IEEE Asia Pacific Conference on Circuits and Systems, pp. 361-364, Seoul, Korea, November 1996.

[32] J. P. Noonan and P. Natarajan, "A general formulation for iterative restoration methods," IEEE Transactions on Signal Processing, vol. 45, no. 10, pp. 2590-2593, 1997.

[33] S. H. Lee, N. I. Cho, and J.-I. Park, "Directional regularisation for constrained iterative image restoration," Electronics Letters, vol. 39, no. 23, pp. 1642-1643, 2003.

[34] W. Chen, M. Chen, and J. Zhou, "Adaptively regularized constrained total least-squares image restoration," IEEE Transactions on Image Processing, vol. 9, no. 4, pp. 588-596, 2000.

[35] S. O. Choy, Y. H. Chan, and W. C. Siu, "Image restoration by regularisation in uncorrelated transform domain," IEE Proceedings: Vision, Image and Signal Processing, vol. 147, no. 6, pp. 587-594, 2000. 
[36] G. Cisneros, E. Bernués, I. Rodríguez, M. A. Santiago, and F. Álvarez, "Desensitisation of medical images restoration under crude estimates of mobile radio channels," in Proceedings of IEEE International Conference on Image Processing (ICIP '04), vol. 1, pp. 315-319, Singapore, October 2004.

[37] R. Molina, A. K. Katsaggelos, and J. Mateos, "Bayesian and regularization methods for hyperparameter estimation in image restoration," IEEE Transactions on Image Processing, vol. 8, no. 2, pp. 231-246, 1999.

[38] R. Molina, J. Mateos, and A. K. Katsaggelos, "Blind deconvolution using a variational approach to parameter, image, and blur estimation," IEEE Transactions on Image Processing, vol. 15, no. 12, pp. 3715-3727, 2006.

[39] R. M. Corless, G. H. Gonnet, D. E. G. Hare, D. J. Jeffrey, and D. E. Knuth, "On the Lambert W function," Advances in Computational Mathematics, vol. 5, no. 4, pp. 329-359, 1996.

Miguel A. Santiago was born in Madrid, Spain, in 1979. He received his degree in telecommunication engineering with honours in 2003 from the Polytechnic University of Madrid (UPM) where he is currently doing his $\mathrm{Ph} . \mathrm{D}$. studies on digital image processing. Since 2003, he has been working as a Technological Consultant of Telefónica of Spain on geographical information systems (GIS) applied on telecommunication

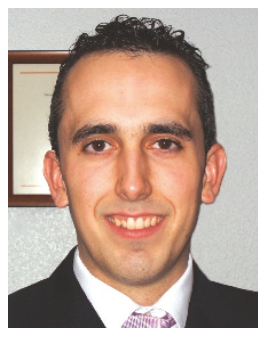
networks with analysis, design, and development activities. He has also been an Active Participant in several European Projects as a Research Associate at the UPM mainly in the areas related to digital television, broadcast, and interactive services. He is collaborating in the IST-027124 project ARENA at present. His main research interests include image restoration, computer vision, and audiovisual applications.

Guillermo Cisneros was born in Madrid (Spain) in 1960. He is a Full Professor of Telecommunication Systems, lecturing on multimedia networks, systems and services, digital image processing, image coding and analysis, and computer graphics. He had the degree of Telecom Engineer with honours since 1983 and Ph.D. in communication engineering cum laude since 1986, both from Universidad Politécnica de Madrid. Besides

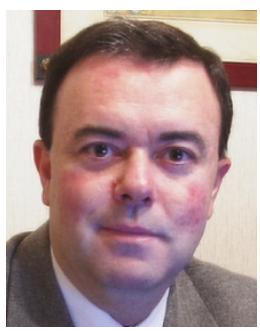
of his lecturing and research activities at the University, he joined Telefónica of Spain for a decade working in technical, managerial, and international business responsibilities in research and mobile communications. He was Spanish Representative in different International Organisations and Working Groups, such as IWP 11/4 of the former CCIR (now UIT-R), GSM, and MoU for GSM establishment. He has also been an Active Participant in several European Projects of different EU Programmes, with strong technological and consortium managerial responsibilities, mainly in the areas related to networked electronic media, digital TV networks, broadcast and interactive services in ubiquitous environments, systems and services for cultural heritage and teleducation applications. He also has been working as an Expert to the European Commission in evaluation and auditing processes, as well as for national Spanish Programmes. Currently, he is Director (Dean) of E.T.S. Ingenieros de Telecomunicación of Universidad Politécnica de Madrid.
Emiliano Bernués received the Master (sixyear course) degree in electrical engineering from the Polytechnic University of Madrid, Spain, in 1993. In 2001, he completed the Ph.D. degree in electrical engineering at the University of Zaragoza, Spain. He is a Professor at the Department of Electronics and Communications Engineering of the University of Zaragoza and carries out his research activities in the Centro Politecnico

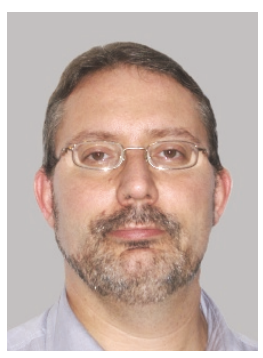
Superior. His research interests are in the area of digital image processing, image restoration, television applications and biometric systems for communication. He has participated in several national and international projects. He has published about 20 papers in international journals and conferences. Currently, he is employed at the Corporation of Radio and Television of Aragon. 